\title{
Transverse dynamics considerations for microbunched electron cooling
}

\author{
P. Baxevanis and G. Stupakov \\ SLAC National Accelerator Laboratory, Menlo Park, California 94025, USA
}

(Received 16 April 2019; published 23 August 2019)

\begin{abstract}
The technique of microbunched electron cooling (MBEC) is an attractive coherent cooling scheme with potential applications in future high-energy circular colliders. In our previous work, we analyzed the cooling of the energy spread using a one-dimensional (1D) technique that tracks the dynamics of microscopic fluctuations in the hadron and electron beams. In this paper, we extend this approach so that it covers the transverse emittance cooling as well. In order to do so, it is necessary to consider the betatron motion of the hadron beam and take into account effects of the momentum dispersion in the modulator and kicker regions. We derive relatively simple analytical expressions for the emittance and energy spread cooling times in terms of the various beam and lattice parameters, allowing us to perform fast optimization studies for an MBEC configuration. Verified through comparison with simulation, our theory can also incorporate features such as plasma amplification stages, which are crucial components of a realistic cooling system.
\end{abstract}

DOI: 10.1103/PhysRevAccelBeams.22.081003

\section{INTRODUCTION}

The technique of microbunched electron cooling (MBEC), first proposed in Ref. [1], is a promising novel scheme for enhancing the brightness of stored hadron beams in future high-energy circular colliders. This requirement is a crucial one for achieving the high luminosities needed for experiments in prospective hadron-hadron and electron-ion machines. In common with other coherent cooling schemes [2,3], in MBEC, the hadron beam first imprints an energy modulation on a copropagating (cooler) electron beam in a segment of the machine known as the modulator. This energy modulation is then converted into a density modulation (bunching) after the e-beam passes through a dispersive chicane section with strength $R_{56}^{(e)}$ (Fig. 1). In the meantime, the hadrons are transported through their own - separate - section of the lattice, which also includes a chicane with strength $R_{56}^{(h)}$. The bunched electron beam then once again interacts with the hadrons in a subsequent section of the machine (the kicker), in a way that can ultimately lead to a significant reduction in the hadron energy spread and transverse emittance after many passages through the cooling section. In order to accelerate this process and ensure that the cooling timescale is small enough for practical purposes, additional amplification stages are typically required, in which the bunching of

Published by the American Physical Society under the terms of the Creative Commons Attribution 4.0 International license. Further distribution of this work must maintain attribution to the author(s) and the published article's title, journal citation, and DOI. the electron beam is boosted through the space charge (or plasma) effect. The latter feature is the main difference between MBEC and other contemporary iterations of coherent cooling, which typically rely on a narrow-band gain mechanism in order to enhance the bunching (for instance, the coherent cooling scheme of Ref. [3] utilizes a free electron laser for this purpose, while that of [4] uses a parametric resonance).

In our previous work [5,6], we presented a onedimensional (1D) theory for MBEC that described the cooling of the hadron energy spread. Our analysis, which only considered the longitudinal portion of the motion, was based on a kinetic theory approach that tracks the evolution of fluctuations in the hadron and electron beams as they move through the cooling lattice. In particular, even though both beams start from a state of uncorrelated shot noise, their propagation develops correlations that can significantly alter the value of macroscopic properties such as the energy spread, leading to cooling. In this paper, we extend this technique so that it can also deal with the mechanism of transverse emittance cooling. In order to achieve this, it is necessary to consider the transverse motion of the hadron beam, including the momentum dispersion, whose interplay with the longitudinal dynamics is the root cause of transverse cooling. We note, however, that our treatment is

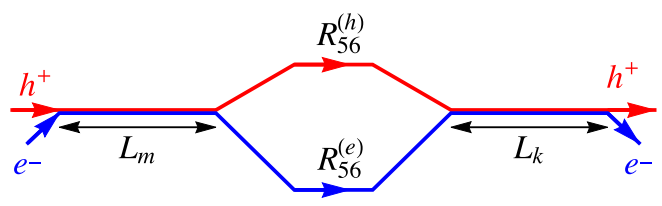

FIG. 1. Schematic of a simple MBEC configuration. 
by no means a fully three-dimensional one: the other essential components of our analysis, such as the motion of the electron beam and the space charge effect, are still being studied in a 1D context. Our main objective is to derive usable analytical expressions for the cooling times of the emittance and energy spread, which would enhance our understanding of the underlying physics and facilitate simple optimization studies.

This paper is organized as follows: in Sec. II, we introduce the basic concepts regarding the betatron motion of the hadrons (including the action-angle variables) and review the description of the hadron shot noise in terms of the fluctuation part of the beam distribution function. In Sec. III, we study the evolution of fluctuations in the hadron beam as the latter passes through the various stages of the cooling system. The influence of the cooler electrons is quantified by the space charge impedance, which-to a large extent-is already known from our previous analysis. In Sec. IV, the results of this study lead us to the formulation of a kinetic equation for the averaged distribution function of the hadrons, derived in the limit of small chicane strength and weak vertical dispersion. For this simplified case, we can show that the hadron energy spread and emittance follow simple exponential decay laws. The assumption of small dispersion and hadron chicane strength is dropped in Sec. V, which derives a generalized version of the kinetic equation. In Sec. VI, we utilize the abovementioned equation and its corollaries in order to obtain analytical formulas that express the transverse and longitudinal cooling times as functions of the various system properties. Using these formulas, we optimize a simple MBEC configuration for the parameters of the proposed eRHIC electron-ion collider. In Sec. VII, we benchmark our analytical expressions by comparing their predictions to the output of a simple simulation algorithm, showing good agreement between the two. In Sec. VIII, we discuss how to include plasma amplification stages in our formalism, outlining the modified expressions for the cooling timescales and providing an updated numerical estimate for eRHIC. Finally, Sec. IX summarizes the basic conclusions of our study. We use the Gaussian system of units throughout this paper.

\section{HADRON BEAM SHOT NOISE}

In our previous work $[5,6]$, we studied one-dimensional (1D) shot noise effects in a particle beam by examining the fluctuations in the beam distribution function. Here, we extend this technique by also considering the transverse motion of the beam, which was neglected in our earlier treatment. Doing so is essential when we seek to describe the mechanism of hadron transverse emittance cooling in MBEC, a process which cannot be analyzed in a purely one-dimensional fashion. That being said, we stress that the motion of the cooler electron beam and the space charge interaction in the modulator, plasma and kicker sections are still being treated in the 1D limit. To start with, we introduce some key variables related to the betatron motion of the hadron beam, as the latter is transported from the modulator to the kicker. Specifically, we need to define the action-angle variables $(J, \phi)$ in terms of the original betatron coordinates $\left(y, P=y^{\prime}=d y / d s\right)$, where $y$ is the vertical displacement and $s$ refers to the longitudinal coordinate along the ring. To simplify our analysis, we only consider the vertical motion of the beam (i.e., the motion in the $y$-direction), neglecting its horizontal counterpart. The action variable $J$ is given by

$$
\begin{aligned}
J= & \frac{1}{2 \beta(s)}\left([y-\eta D(s)]^{2}\right. \\
& \left.+\left\{\beta(s)\left[P-\eta D^{\prime}(s)\right]+\alpha(s)[y-\eta D(s)]\right\}^{2}\right),
\end{aligned}
$$

where $\beta(s)$ and $\alpha(s)=-\beta^{\prime}(s) / 2$ are the standard CourantSnyder parameters, $D(s)$ is the vertical dispersion, and $\eta=$ $\Delta E / E_{0}$ is the relative energy deviation of a hadron with respect to the nominal energy $E_{0}=\gamma m_{h} c^{2}$. Here, we clarify that the prime over the lattice functions denotes differentiation with respect to $s$, just like in $y^{\prime}$. For pure betatron motion (that is, in the absence of any effects that can change the hadron energy), $J$ is a constant of the motion. For the betatron angle $\phi$, we have the relation

$$
\cos \phi=\frac{y-\eta D(s)}{\sqrt{2 \beta(s) J}}
$$

with $d \phi(s) / d s=1 / \beta(s)$. We also require the relations that express the original coordinates in terms of the action-angle variables, namely

$$
\begin{aligned}
& y=\eta D(s)+\sqrt{2 \beta(s) J} \cos \phi, \\
& P=\frac{d y}{d s}=\eta D^{\prime}(s)-\sqrt{\frac{2 J}{\beta(s)}}(\sin \phi+\alpha(s) \cos \phi) .
\end{aligned}
$$

The initial distribution function, which describes the state of the hadron beam before the latter enters the modulator section, can be written as

$$
f_{\text {init }}(z, \eta, J, \phi)=n_{0 h} F_{h}(\eta, J)+\delta f(z, \eta, J, \phi),
$$

where, in keeping with our earlier notation, $z=s-v_{0} t$ refers to the longitudinal coordinate inside the bunch $\left[v_{0}=c\left(1-\gamma^{-2}\right)^{1 / 2}\right.$ is the nominal velocity]. Additionally, $n_{0 h}$ is the linear density of the hadron beam (number of particles per unit length), $F_{h}$ is the equilibrium beam distribution and $\delta f$ is the fluctuation part that contains the shot noise. Here, we assume that the equilibrium hadron distribution $F_{h}(\eta, J)$ does not depend on the internal bunch position $z$-an approximation which is justified by the fact that we only consider fluctuations on a scale that is much 
smaller than the bunch length. Moreover, $F_{h}$ does not depend on the betatron angle $\phi$; this reflects the fact that the cooling timescale is much larger than the revolution period of the ring. As a result, any $\phi$-dependence is eventually washed out over multiple turns. Lastly, the normalization of $F_{h}$ is defined by

$$
\begin{aligned}
& \int_{-\infty}^{\infty} d \eta \int_{0}^{\infty} d J \int_{0}^{2 \pi} d \phi F_{h}(\eta, J) \\
& =2 \pi \int_{-\infty}^{\infty} d \eta \int_{0}^{\infty} d J F_{h}(\eta, J)=1 .
\end{aligned}
$$

The function $\delta f(z, \eta, J, \phi)$ describes the statistical fluctuations in the hadron beam and has an average value equal to zero, so that $\langle\delta f(z, \eta, J, \phi)\rangle=0$, where the brackets denote ensemble averaging. It is worth noting that, in order to simplify our notation, we have dropped the independent variable $s$ from the arguments of $\delta f$ as we will only be calculating $\delta f$ at specific locations along the MBEC lattice. The one-dimensional Fourier transformation of $\delta f$ is defined by

$$
\begin{aligned}
\delta \hat{f}_{k}(\eta, J, \phi) & =\int_{-\infty}^{\infty} d z e^{-i k z} \delta f(z, \eta, J, \phi), \\
\delta f(z, \eta, J, \phi) & =\frac{1}{2 \pi} \int_{-\infty}^{\infty} d k e^{i k z} \delta \hat{f}_{k}(\eta, J, \phi) .
\end{aligned}
$$

Neglecting the interaction between the hadrons, the correlator for the random fluctuation part $\delta f$ at two different points in phase space is given by

$$
\begin{aligned}
& \left\langle\delta f(z, \eta, J, \phi) \delta f\left(z^{\prime}, \eta^{\prime}, J^{\prime}, \phi^{\prime}\right)\right\rangle \\
& \quad=n_{0 h} F_{h}(\eta, J) \delta\left(z-z^{\prime}\right) \delta\left(\eta-\eta^{\prime}\right) \delta\left(J-J^{\prime}\right) \delta\left(\phi-\phi^{\prime}\right) .
\end{aligned}
$$

The above relation is the basic result governing the initial shot noise in the hadron beam. A corresponding relation for $\delta \hat{f}_{k}$ is

$$
\begin{aligned}
& \left\langle\delta \hat{f}_{k}(\eta, J, \phi) \delta \hat{f}_{k^{\prime}}\left(\eta^{\prime}, J^{\prime}, \phi^{\prime}\right)\right\rangle \\
& =2 \pi n_{0 h} F_{h}(\eta, J) \delta\left(k+k^{\prime}\right) \delta\left(\eta-\eta^{\prime}\right) \delta\left(J-J^{\prime}\right) \delta\left(\phi-\phi^{\prime}\right) .
\end{aligned}
$$

Introducing the density fluctuation

$$
\delta n(z)=\int_{-\infty}^{\infty} d \eta \int_{0}^{\infty} d J \int_{0}^{2 \pi} d \phi \delta f(z, \eta, J, \phi),
$$

one can readily show that

$$
\left\langle\delta n(z) \delta n\left(z^{\prime}\right)\right\rangle=n_{0 h} \delta\left(z-z^{\prime}\right),
$$

which is identical to the result of the one-dimensional analysis [5]. Another important quantity is the Fourier spectrum of $\delta n(z)$, which is given by

$$
\begin{aligned}
\delta \hat{n}_{k} & =\int_{-\infty}^{\infty} d z e^{-i k z} \delta n(z) \\
& =\int_{-\infty}^{\infty} d \eta \int_{0}^{\infty} d J \int_{0}^{2 \pi} d \phi \delta \hat{f}_{k}(\eta, J, \phi) .
\end{aligned}
$$

The correlator for this new variable is simply

$$
\left\langle\delta \hat{n}_{k} \delta \hat{n}_{k^{\prime}}\right\rangle=2 \pi n_{0 h} \delta\left(k+k^{\prime}\right) .
$$

Finally, by integrating Eq. (7) over $\eta^{\prime}, J^{\prime}$ and $\phi^{\prime}$ and taking the Fourier transform over $z^{\prime}$, we obtain the following result (frequently used in Appendixes A and B):

$$
\left\langle\delta f(z, \eta, J, \phi) \delta \hat{n}_{k}\right\rangle=n_{0 h} F_{h}(\eta, J) e^{-i k z} .
$$

\section{DYNAMICS OF FLUCTUATIONS IN THE HADRON BEAM}

Propagating the hadron beam through the cooling section results in a modification of its input distribution function, a process which can lead to a cooling effect (for both the emittance and the energy spread) after many revolutions. In this section, we seek to quantify this modification by tracking the evolution of the hadron fluctuations from the modulator to the kicker. To start with, we assume that the initial distribution function of the hadrons is given by Eq. (4) and that the beam is in a state with uncorrelated shot noise, as described by Eq. (7). To distinguish between the initial fluctuation part of the distribution function and its final counterpart, we will change the notation $\delta f$ in Eq. (4) to $\delta f^{(M)}$, where $M$ refers to the modulator. The hadron beam first interacts with the electrons in the aforementioned section, where each hadron creates a perturbation in the electron beam. This perturbation is localized in the immediate vicinity of the hadron. Strictly speaking, electrons also perturb the hadron beam during this interaction, but we will neglect this effect in our analysis. The energy modulation imprinted on the electrons in the modulator, like the subsequent kick that they impart on the hadrons in the kicker, is the same as in our original 1D model. This is so because both of these energy changes are produced by longitudinal density fluctuations, on which transverse effects such as dispersion have no big impact (apart from modifying the beam cross section). Moreover, we assume that the modulator and kicker lengths are relatively small, so that the lattice functions $\alpha, \beta$ and $D$-as well as the orbit coordinate $y$ and its slope $y^{\prime}=$ $P$-can be treated as constants for these two segments. Thus, the vector $(y, P, z, \eta)^{T}$, which contains the phase space coordinates of a single hadron, is not changed by passage through the modulator and neither is the hadron distribution function (here, $T$ denotes the transpose).

On the other hand, tracking the distribution function through the hadron transfer line between the modulator and the kicker requires a more involved analysis. Since we are 
primarily interested in the interplay between the longitudinal and the vertical degrees of freedom (d.o.f.), we need to define a four-dimensional transport matrix $R_{t}$ for the hadrons, which is given by

$$
R_{t}=\left(\begin{array}{cccc}
R_{33} & R_{34} & 0 & R_{36} \\
R_{43} & R_{44} & 0 & R_{46} \\
R_{53} & R_{54} & 1 & R_{56}^{(h)} \\
0 & 0 & 0 & 1
\end{array}\right) .
$$

This matrix acts on the coordinate vector $(y, P, z, \eta)^{T}$ and propagates it from the end of the modulator section to the start of the kicker. A useful set of general expressions for the various matrix elements is given in Ref. [7]:

$$
\begin{aligned}
& R_{33}=\sqrt{\frac{\beta_{2}}{\beta_{1}}}\left(\cos \mu+\alpha_{1} \sin \mu\right), \quad R_{34}=\sqrt{\beta_{1} \beta_{2}} \sin \mu, \\
& R_{43}=\frac{\alpha_{1}-\alpha_{2}}{\sqrt{\beta_{1} \beta_{2}}} \cos \mu-\frac{1+\alpha_{1} \alpha_{2}}{\sqrt{\beta_{1} \beta_{2}}} \sin \mu, \\
& R_{44}=\sqrt{\frac{\beta_{1}}{\beta_{2}}}\left(\cos \mu-\alpha_{2} \sin \mu\right),
\end{aligned}
$$

and

$$
\begin{aligned}
& R_{36}=D_{2}-R_{33} D_{1}-R_{34} D_{1}^{\prime} \\
& R_{46}=D_{2}^{\prime}-R_{43} D_{1}-R_{44} D_{1}^{\prime} \\
& R_{53}=R_{43} R_{36}-R_{33} R_{46} \\
& R_{54}=R_{44} R_{36}-R_{34} R_{46} .
\end{aligned}
$$

Here, the subscripts $1 / 2$ refer to the beginning/end of the hadron transfer line (respectively) while $\mu=\phi_{2}-\phi_{1}$ is the total phase advance for the same segment. We note that, in the absence of dispersion, the matrix $R_{t}$ becomes blockdiagonal and no coupling exists between the transverse and longitudinal d.o.f. In addition, we emphasize that $R_{t}$ is a symplectic matrix, i.e., it satisfies the equality $R_{t}^{T} \Omega R_{t}=\Omega$, with

$$
\Omega=\left(\begin{array}{cccc}
0 & 1 & 0 & 0 \\
-1 & 0 & 0 & 0 \\
0 & 0 & 0 & 1 \\
0 & 0 & -1 & 0
\end{array}\right)
$$

Making use of this property is necessary when one seeks to derive the analytical results of Eqs. (15)-(16). Starting from first principles, this calculation also utilizes Eq. (3).

The passage through the transfer line with transport matrix $R_{t}$ introduces a phase space transformation $\left(z_{1}, \eta_{1}, J_{1}, \phi_{1}\right) \rightarrow\left(z_{2}, \eta_{2}, J_{2}, \phi_{2}\right)$, with $\eta_{2}=\eta_{1}, J_{2}=J_{1}$, $\phi_{2}=\phi_{1}+\mu$ and

$$
z_{2}=z_{1}+R_{53} y_{1}+R_{54} P_{1}+R_{56}^{(h)} \eta_{1}
$$

This changes the initial hadron distribution function $f_{\text {init }}$ in the modulator into a different function $f_{2}$ at the start of the kicker, so that $f_{\text {init }}=f_{1} \rightarrow f_{2}$. The new distribution function is obtained by expressing the old arguments in terms of the new ones. ${ }^{1}$ For this, we need the backward transformation from the kicker to the modulator, which is given by the inverse matrix $\hat{R}_{t} \equiv R_{t}^{-1}$. Using the symplectic character of $R_{t}$, we can show that $\hat{R}_{t}=-\Omega R_{t}^{T} \Omega$. Calculating $\hat{R}_{t}$ and then multiplying this matrix by $\left(y_{2}, P_{2}, z_{2}, \eta_{2}\right)^{T}$, one finds that

$$
\begin{aligned}
z_{1}= & z_{2}+R_{46} y_{2}-R_{36} P_{2}-R_{56}^{(h)} \eta_{2} \\
= & z_{2}+R_{46}\left(\eta_{2} D_{2}+\sqrt{2 \beta_{2} J} \cos \phi_{2}\right) \\
& -R_{36}\left(\eta_{2} D_{2}^{\prime}-\sqrt{\frac{2 J}{\beta_{2}}}\left(\sin \phi_{2}+\alpha_{2} \cos \phi_{2}\right)\right)-R_{56}^{(h)} \eta_{2} .
\end{aligned}
$$

Thus, the new hadron distribution function is

$$
\begin{aligned}
f_{2}(z, \eta, J, \phi)= & f_{\text {init }}\left(z+R_{46} y-R_{36} P-R_{56}^{(h)} \eta, \eta, J, \phi-\mu\right) \\
= & n_{0 h} F_{h}(\eta, J)+\delta f^{(M)}\left(z+R_{46} y-R_{36} P\right. \\
& \left.-R_{56}^{(h)} \eta, \eta, J, \phi-\mu\right),
\end{aligned}
$$

where we have made use of Eq. (19). We note that we have dropped the index 2 in the notation for the variables $z, y, P$ and $\phi$ - the fact that this distribution function refers to the start of the kicker region is now indicated by indexing the function $f$. In this expression, $y$ and $P$ are understood as functions of $J, \phi$, and $\eta$, as shown in Eqs. (3). In particular, we note that the important quantity $Q=R_{46} y-R_{36} P-R_{56}^{(h)} \eta$ can be written as

$$
\begin{aligned}
Q(\eta, J, \phi) & =-Q_{0} \eta+\bar{Q}(J, \phi) \\
& =-Q_{0} \eta+\sqrt{J}\left(Q_{A} \cos \phi+Q_{B} \sin \phi\right),
\end{aligned}
$$

where

$$
\begin{aligned}
Q_{0} & =R_{56}^{(h)}-R_{46} D_{2}+R_{36} D_{2}^{\prime}, \\
Q_{A} & =\sqrt{2}\left(R_{46} \sqrt{\beta_{2}}+R_{36} \frac{\alpha_{2}}{\sqrt{\beta_{2}}}\right), \\
Q_{B} & =\sqrt{2} \frac{R_{36}}{\sqrt{\beta_{2}}} .
\end{aligned}
$$

\footnotetext{
${ }^{1}$ Here we implicitly assume that the hadron motion from the kicker to the modulator is Hamiltonian. As a result, the value of the distribution function remains constant along individual phase space trajectories.
} 
Lastly, we consider the passage through the kicker section. In the latter, the hadrons change energy due to the interaction with the fluctuational longitudinal electric field of the electron beam, from an initial energy $\eta_{2}$ to a final energy $\eta_{3}$ (so that $\eta_{3}=\eta_{2}+\Delta \eta^{(h)}\left(z_{2}\right)$, where $\Delta \eta^{(h)}$ is the energy change and the index 3 refers to the end of the kicker). Here, we assume that the hadron longitudinal positions are unchanged, $z_{3}=z_{2}$, which is valid for a short kicker segment. This energy variation results in a corresponding change of the action-angle variables: $J_{3}=$ $J_{2}+\Delta J^{(h)}\left(z_{2}, J_{2}, \phi_{2}\right)$ and $\phi_{3}=\phi_{2}+\Delta \phi^{(h)}\left(z_{2}, J_{2}, \phi_{2}\right)$. Analytical expressions for the shifts $\Delta J^{(h)}$ and $\Delta \phi^{(h)}$ can be obtained in the following way: using Eq. (1), one can show that an energy change $\Delta \eta^{(h)}\left(z_{2}\right)$ alters the action variable by an amount equal to

$$
\begin{aligned}
& \Delta \boldsymbol{J}^{(h)}\left(z_{2}, J_{2}, \phi_{2}\right) \\
& =\Delta \eta^{(h)}\left(z_{2}\right) \sqrt{\frac{2 J_{2}}{\beta_{2}}}\left[\left(\beta_{2} D_{2}^{\prime}+\alpha_{2} D_{2}\right) \sin \phi_{2}-D_{2} \cos \phi_{2}\right] \\
& \quad+\frac{\left(\Delta \eta^{(h)}\left(z_{2}\right)\right)^{2}}{2 \beta_{2}}\left[D_{2}^{2}+\left(\beta_{2} D_{2}^{\prime}+\alpha_{2} D_{2}\right)^{2}\right],
\end{aligned}
$$

where we recall that $\alpha, \beta$ and $D$ are treated as constants in the kicker. Note that, in contrast to the energy, action and betatron angle, the longitudinal electric field does not change the offset $y$ and the variable $P$, so $y_{3}=y_{2}$ and $P_{3}=P_{2}$. A result of the synergy between dispersion and energy change, this variation in the action is eventually responsible for the emittance cooling effect. From Eq. (2), the corresponding change in the angle $\phi$ is

$$
\begin{aligned}
& \Delta \phi^{(h)}\left(z_{2}, J_{2}, \phi_{2}\right) \\
& \quad=\frac{\Delta \eta^{(h)}\left(z_{2}\right)}{\sqrt{2 \beta_{2} J_{2}}}\left[D_{2} \sin \phi_{2}+\left(\beta_{2} D_{2}^{\prime}+\alpha_{2} D_{2}\right) \cos \phi_{2}\right] .
\end{aligned}
$$

For the angular shift $\Delta \phi^{(h)}$, it is only necessary to keep terms linear in $\Delta \eta^{(h)}$. For the transformation of the distribution function, we will need to express the initial variables in terms of the final ones, which can only be done in an approximate fashion. Keeping terms up to linear order in $\Delta \eta^{(h)}$ for all variables except the action (for which-as before-we need to go up to second order), the result is

$$
\begin{aligned}
z_{2} & =z_{3}, \\
\eta_{2} & =\eta_{3}-\Delta \eta^{(h)}\left(z_{3}\right), \\
J_{2} & =J_{3}-\Delta \bar{J}^{(h)}\left(z_{3}, J_{3}, \phi_{3}\right), \\
\phi_{2} & =\phi_{3}-\Delta \phi^{(h)}\left(z_{3}, J_{3}, \phi_{3}\right),
\end{aligned}
$$

where

$$
\begin{aligned}
& \Delta \bar{J}^{(h)}(z, J, \phi) \\
& =\Delta \bar{J}^{(h, 1)}(z, J, \phi)+\Delta \bar{J}^{(h, 2)}(z, J, \phi) \\
& \equiv \Delta \eta^{(h)}(z) \sqrt{\frac{2 J}{\beta_{2}}}\left[\left(\beta_{2} D_{2}^{\prime}+\alpha_{2} D_{2}\right) \sin \phi-D_{2} \cos \phi\right] \\
& \quad-\frac{\left[\Delta \eta^{(h)}(z)\right]^{2}}{2 \beta_{2}}\left[D_{2}^{2}+\left(\beta_{2} D_{2}^{\prime}+\alpha_{2} D_{2}\right)^{2}\right],
\end{aligned}
$$

where $\Delta \bar{J}^{(h, 1)}(z, J, \phi)$ is the part that is proportional to $\Delta \eta^{(h)}(z)$ while $\Delta \bar{J}^{(h, 2)}(z, J, \phi)$ is the quadratic term. This transformation results in a new hadron distribution function after the kicker, i.e., $f_{2} \rightarrow f_{3}$, where

$$
\begin{aligned}
f_{3}(z, \eta, J, \phi)= & f_{2}\left(z, \eta-\Delta \eta^{(h)}, J-\Delta \overline{\boldsymbol{J}}^{(h)}, \phi-\Delta \phi^{(h)}\right) \\
= & n_{0 h} F_{h}\left(\eta-\Delta \eta^{(h)}, J-\Delta \overline{\boldsymbol{J}}^{(h)}\right) \\
& +\delta f^{(M)}\left(z+R_{46} y-R_{36} P-R_{56}^{(h)} \eta\right. \\
& +R_{56}^{(h)} \Delta \eta^{(h)}, \eta-\Delta \eta^{(h)}, J-\Delta \bar{J}^{(h)} \\
& \left.\phi-\mu-\Delta \phi^{(h)}\right) .
\end{aligned}
$$

For brevity, in Eq. (27) we have omitted the argument $z$ in the function $\Delta \eta^{(h)}$ and $(z, J, \phi)$ in both $\Delta \bar{J}^{(h)}$ and $\Delta \phi^{(h)}$. An additional clarification is in order regarding a more subtle point of Eq. (27): since the backward transformation of Eq. (25) keeps $y$ and $P$ constant, the shift defined by it only affects $\eta$ out of the three arguments of the quantity $Q(\eta, J, \phi)=R_{46} y-R_{36} P-R_{56}^{(h)} \eta$.

As far as the specific expression for the hadron energy change $\Delta \eta^{(h)}(z)$ is concerned, we can reuse the result derived in our 1D analysis [5], namely

$$
\Delta \eta^{(h)}(z)=-\frac{r_{h} c}{2 \pi \gamma} \int_{-\infty}^{\infty} d k \mathcal{Z}(k) \delta \hat{n}_{k}^{(M)} e^{i k z}
$$

where $\mathcal{Z}(k)$ is the impedance associated with the hadronelectron interaction, $\delta \hat{n}_{k}^{(M)}$ is the Fourier transform of the initial density modulation $\delta n^{(M)}(z)$-obtained by setting $\delta \hat{f}_{k} \rightarrow \delta \hat{f}_{k}^{(M)}$ in Eq. (11)—and $r_{h}=(Z e)^{2} / m_{h} c^{2}$ (where $Z e$ is the hadron charge and $m_{h}$ is the hadron mass). Introducing the Fourier transform

$$
\Delta \hat{\eta}_{k}^{(h)}=\int_{-\infty}^{\infty} d z e^{-i k z} \Delta \eta^{(h)}(z),
$$

we also obtain

$$
\Delta \hat{\eta}_{k}^{(h)}=-\frac{r_{h} c}{\gamma} \mathcal{Z}(k) \delta \hat{n}_{k}^{(M)} .
$$

An analytical formula for the impedance $\mathcal{Z}(k)$ will be introduced in Sec. VI. Even in this general form, Eq. (28) is essential for calculating shot noise-related statistical 
averages (see Appendixes A and B). It is also important to remember that $\delta \hat{n}_{k}^{(M)}$ is associated with the density fluctuations in the modulator-the place where the initial hadron fluctuations are imprinted on the electrons. These fluctuations should be calculated using the initial fluctuation part of the distribution function $\delta f^{(M)}(z, \eta, J, \phi)$. When hadrons arrive at end of the kicker, their distribution function has changed to $f_{3}$, as given by Eq. (27).

\section{COHERENT COOLING IN THE LIMIT OF WEAK DISPERSION AND SMALL HADRON CHICANE STRENGTH}

As we have already seen, the distribution function of the hadron beam is altered as a result of the passage through the cooling section. Introducing the difference

$$
\Delta f=f_{3}(z, \eta, J, \phi)-f_{\text {init }}(z, \eta, J, \phi),
$$

we anticipate that the ensemble-averaged value $\langle\Delta f\rangle$ would be nonzero (but much smaller in absolute value than $\left.n_{0 h} F_{h}\right)$. This behavior is due to transport-related correlations developing in the beam, which shift it away from its initial state of uncorrelated shot noise [see the arguments of $\delta f^{(M)}$ in Eq. (27)]. As in our original 1D analysis, we associate this average value of $\Delta f$ with the change of the equilibrium distribution function $F_{h}$ during one revolution period $T$ :

$$
n_{0 h} T \frac{\partial F_{h}}{\partial t}=\langle\Delta f\rangle
$$

In a manner analogous to the study of synchrotron oscillations in a ring, this partial differential equation describes the temporal evolution of the hadron beam over a timescale that is much larger than $T$. This essentially allows us to replace an iterative map formalism with one that assumes a slow, continuous dependence with respect to $t$.

In what follows, our immediate objective is to calculate $\langle\Delta f\rangle$. To simplify our analysis, we will assume that the longitudinal shift $Q=R_{46} y-R_{36} P-R_{56}^{(h)} \eta$ is small and neglect terms of higher than the first order in this expression. Reviewing Eqs. (15)-(16) and (21)-(22), we can easily establish that $Q$ has a small value when $R_{56}^{(h)}, R_{36}$, and $R_{46}$ are, in turn, small. For the last two matrix elements, this is equivalent to stating that the dispersion is weak. In addition, we will use the smallness of the fluctuations in the beam and treat $\Delta \eta^{(h)}$ and $\delta f^{(M)}$ as small quantities as well $(\sim \mathcal{E})$, neglecting terms of order $\varepsilon^{3}$ and higher. Using the Taylor expansion in terms of the parameter $\varepsilon$ (and recalling the decomposition of the action shift given by Eq. (26), we obtain

$$
\begin{aligned}
\langle\Delta f\rangle \approx- & n_{0 h}\left\langle\Delta \bar{J}^{(h, 2)}\right\rangle \partial_{J} F_{h}+\frac{1}{2} n_{0 h}\left\langle\left(\Delta \eta^{(h)}\right)^{2}\right\rangle \partial_{\eta \eta} F_{h}+n_{0 h}\left\langle\Delta \eta^{(h)} \Delta \bar{J}^{(h, 1)}\right\rangle \partial_{\eta J} F_{h}+\frac{1}{2} n_{0 h}\left\langle\left(\Delta \bar{J}^{(h, 1)}\right)^{2}\right\rangle \partial_{J J} F_{h} \\
& -\left\langle\Delta \eta^{(h)} \partial_{\eta} \delta f^{(M)}\right\rangle-\left\langle\Delta \bar{J}^{(h, 1)} \partial_{J} \delta f^{(M)}\right\rangle-\left\langle\Delta \phi^{(h)} \partial_{\phi} \delta f^{(M)}\right\rangle-\left(R_{46} y-R_{36} P-R_{56}^{(h)} \eta\right)\left\langle\Delta \eta^{(h)} \partial_{z \eta} \delta f^{(M)}\right\rangle \\
& -\left(R_{46} y-R_{36} P-R_{56}^{(h)} \eta\right)\left\langle\Delta \bar{J}^{(h, 1)} \partial_{z J} \delta f^{(M)}\right\rangle-\left(R_{46} y-R_{36} P-R_{56}^{(h)} \eta\right)\left\langle\Delta \phi^{(h)} \partial_{z \phi} \delta f^{(M)}\right\rangle \\
& +R_{56}^{(h)}\left\langle\Delta \eta^{(h)} \partial_{z} \delta f^{(M)}\right\rangle,
\end{aligned}
$$

where, for purposes of notational simplicity, we have omitted the arguments $\eta, J$ in the function $F_{h}$ and $z, \eta, J, \phi-\mu$ in $\delta f^{(M)}$. In obtaining the result given above, we have used the fact that $\left\langle\delta f^{(M)}\right\rangle=0$ and $\left\langle\Delta \eta^{(h)}\right\rangle=$ $\left\langle\Delta \bar{J}^{(h, 1)}\right\rangle=\left\langle\Delta \phi^{(h)}\right\rangle=0$. The last (triple) equality can be justified as follows: taking the Fourier transform of the relation $\left\langle\delta f^{(M)}\right\rangle=0$, we obtain $\left\langle\delta \hat{f}_{k}^{(M)}\right\rangle=0$. In view of Eqs. (11) and (28), this implies that $\left\langle\delta \hat{n}_{k}^{(M)}\right\rangle=0$ and $\left\langle\Delta \eta^{(h)}\right\rangle=0$. Since $\Delta \overline{\boldsymbol{J}}^{(h, 1)}$ and $\Delta \phi^{(h)}$ are both proportional to $\Delta \eta^{(h)}$ [see Eqs. (26) and (24)], the remaining two equations follow directly.

Calculating all the terms on the right-hand side (RHS) of Eqs. (33) is a lengthy analytical exercise that is carried out in Appendix A. Here, we only state the final result, which is a kinetic equation for the averaged distribution function $F_{h}$ :

$$
\begin{aligned}
\frac{\partial F_{h}}{\partial t}= & D_{\eta} \frac{\partial^{2} F_{h}}{\partial \eta^{2}}+D_{\epsilon} \frac{\partial}{\partial J}\left(J \frac{\partial F_{h}}{\partial J}\right) \\
& +w^{\prime}(0) \frac{r_{h}}{T \gamma}\left[-S \frac{\partial\left(J F_{h}\right)}{\partial J}+\left(S-R_{56}^{(h)}\right) \frac{\partial\left(\eta F_{h}\right)}{\partial \eta}\right]
\end{aligned}
$$

where $S \equiv R_{46} D_{2}-R_{36} D_{2}^{\prime}, w^{\prime}(0)$ is the value of the wake derivative $d w(z) / d z$ at $z=0$ (the wake function $w(z)$ being defined in Eq. (A8) in terms of the impedance) and

$$
\begin{aligned}
D_{\eta} & =\frac{n_{0 h}}{4 \pi T}\left(\frac{r_{h} c}{\gamma}\right)^{2} \int_{-\infty}^{\infty} d k|\mathcal{Z}(k)|^{2}, \\
D_{\epsilon} & =\frac{D_{2}^{2}+\left(\beta_{2} D_{2}^{\prime}+\alpha_{2} D_{2}\right)^{2}}{\beta_{2}} D_{\eta},
\end{aligned}
$$

are the diffusion coefficients. The latter are labeled so because the first two terms on the RHS of Eq. (34) describe the diffusion, whereas the remaining three terms 
describe the cooling. Here, we must clarify that we have neglected the contribution due to the wake at the origin given by Eq. (A7), which would have added a term equal to $-\left(r_{h} / T \gamma\right) w(0) \partial_{\eta} F_{h}=\left(r_{h} c / 2 \pi T \gamma\right)\left[\int_{-\infty}^{\infty} d k \mathcal{Z}(k)\right] \partial_{\eta} F_{h}$ to the RHS of Eq. (34).

The first thing one has to verify is that Eq. (34) conserves particles. For this we integrate both sides of the kinetic equation by $\int_{-\infty}^{\infty} d \eta \int_{0}^{\infty} d J$. After integration by parts, we indeed find that the total number of particles is conserved, i.e.,

$$
\frac{d}{d t} \int_{-\infty}^{\infty} d \eta \int_{0}^{\infty} d J F_{h}=0
$$

Next, we calculate the rate of change for the beam energy spread $\sigma_{\eta}$ and the transverse emittance $\epsilon$, which are defined by

$$
\begin{gathered}
\sigma_{h}^{2}=2 \pi \int_{-\infty}^{\infty} d \eta \int_{0}^{\infty} d J \eta^{2} F_{h}, \\
\epsilon=2 \pi \int_{-\infty}^{\infty} d \eta \int_{0}^{\infty} d J J F_{h} .
\end{gathered}
$$

Here, the $2 \pi$ factor comes from the normalization given by Eq. (5). Combining Eq. (34) with the definitions given above, we obtain

$$
\begin{aligned}
\frac{d \sigma_{h}^{2}}{d t} & =-2 w^{\prime}(0) \frac{r_{h}}{T \gamma}\left(S-R_{56}^{(h)}\right) \sigma_{h}^{2}+2 D_{\eta}=-\frac{\sigma_{h}^{2}}{N_{c}^{\eta} T}+2 D_{\eta}, \\
\frac{d \epsilon}{d t} & =w^{\prime}(0) \frac{r_{h}}{T \gamma} S \epsilon+D_{\epsilon}=-\frac{\epsilon}{N_{c}^{\epsilon} T}+D_{\epsilon},
\end{aligned}
$$

where we have introduced the cooling times for energy spread and emittance $\left(N_{c}^{\eta}\right.$ and $\left.N_{c}^{\epsilon}\right)$ in terms of the revolution period $T$. Neglecting the diffusion effects quantified by $D_{\eta}$ and $D_{\epsilon}$, we can see that simultaneous exponential cooling of the energy spread and the emittance is possible if $w^{\prime}(0) S<0$ and $w^{\prime}(0)\left(S-R_{56}^{(h)}\right)>0$. The effect of the dispersion is contained in the parameter $S=R_{46} D_{2}-R_{36} D_{2}^{\prime}$. Using
Eqs. (15) and (16), we obtain a general expression for this quantity, namely

$$
\begin{aligned}
S= & \frac{D_{1} D_{2}}{\sqrt{\beta_{1} \beta_{2}}}\left[\left(\alpha_{1} \alpha_{2}+1\right) \sin \mu+\left(\alpha_{2}-\alpha_{1}\right) \cos \mu\right] \\
& +D_{1} D_{2}^{\prime} \sqrt{\frac{\beta_{2}}{\beta_{1}}}\left(\alpha_{1} \sin \mu+\cos \mu\right) \\
& -D_{1}^{\prime} D_{2} \sqrt{\frac{\beta_{1}}{\beta_{2}}}\left(\cos \mu-\alpha_{2} \sin \mu\right)+D_{1}^{\prime} D_{2}^{\prime} \sqrt{\beta_{1} \beta_{2}} \sin \mu .
\end{aligned}
$$

This is in agreement with Ref. [7]. In the absence of dispersion, $S=0$ and only cooling of the energy spread is possible (which is essentially the 1D case studied before). It is also worth noting that the scaled cooling rates satisfy the relation

$$
\frac{1}{2 N_{c}^{\eta}}+\frac{1}{N_{c}^{\epsilon}}=-w^{\prime}(0) \frac{r_{h}}{\gamma} R_{56}^{(h)},
$$

the RHS of which does not depend on the dispersion (provided we neglect a small, quadratic dependence of the wake with respect to the dispersion). On the other hand, the ratio of the rates is given by $N_{c}^{\epsilon} / N_{c}^{\eta}=2\left(R_{56}^{(h)}-S\right) / S$.

\section{ANALYSIS FOR ARBITRARY VALUES OF THE MATRIX ELEMENTS}

In this section, we generalize the kinetic equation for the hadron distribution function $F_{h}$ by removing the assumption about the smallness of the various transport matrix elements (namely $R_{36}, R_{46}$, and $R_{56}^{(h)}$ ). This generalization does not change the expressions for the diffusion terms but it does modify the calculation for the cooling terms. The details of this updated derivation are contained in Appendix B. After going through it, we find that the new kinetic equation is

$$
\begin{aligned}
\frac{\partial F_{h}}{\partial t}= & D_{\eta} \frac{\partial^{2} F_{h}}{\partial \eta^{2}}+D_{\epsilon} \frac{\partial}{\partial J}\left(J \frac{\partial F_{h}}{\partial J}\right)+\frac{r_{h} c}{2 \pi \gamma T} \frac{\partial}{\partial \eta}\left(F_{h} \int_{-\infty}^{\infty} d k \mathcal{Z}(k)\left\{e^{i k Q_{0} \eta} J_{0}(k R \sqrt{J})-1\right\}\right) \\
& +\frac{r_{h} c}{2 \pi \gamma T} i S \frac{\partial}{\partial J}\left(F_{h} \int_{-\infty}^{\infty} d k \mathcal{Z}(k) e^{i k Q_{0} \eta} \frac{2 \sqrt{J} J_{1}(k R \sqrt{J})}{R}\right),
\end{aligned}
$$

where we have included the diffusion terms from Eq. (34) and (again) subtracted the effect of the wake at the origin. Additionally, we clarify that $J_{n}$ are the Bessel functions of the first kind and recall that $Q_{0}=R_{56}^{(h)}-S$. An expression for the important new parameter $R=\left(Q_{A}^{2}+Q_{B}^{2}\right)^{1 / 2}$ can be obtained using Eqs. (15), (16), and (22). The end result is

$$
\begin{aligned}
R^{2}= & 2 \frac{1+\alpha_{1}^{2}}{\beta_{1}} D_{1}^{2}+2 \frac{1+\alpha_{2}^{2}}{\beta_{2}} D_{2}^{2}-4 \frac{\left(1+\alpha_{1} \alpha_{2}\right) \cos \mu+\left(\alpha_{1}-\alpha_{2}\right) \sin \mu}{\sqrt{\beta_{1} \beta_{2}}} D_{1} D_{2}+2 \beta_{1} D_{1}^{\prime 2}-4 \sqrt{\beta_{1} \beta_{2}} \cos \mu D_{1}^{\prime} D_{2}^{\prime}+2 \beta_{2} D_{2}^{\prime 2} \\
& +4 \alpha_{1} D_{1} D_{1}^{\prime}+4 \alpha_{2} D_{2} D_{2}^{\prime}+4 \sqrt{\frac{\beta_{2}}{\beta_{1}}}\left(\sin \mu-\alpha_{1} \cos \mu\right) D_{1} D_{2}^{\prime}-4 \sqrt{\frac{\beta_{1}}{\beta_{2}}}\left(\sin \mu+\alpha_{2} \cos \mu\right) D_{2} D_{1}^{\prime} .
\end{aligned}
$$


In the limit of small $R$ and $Q_{0}$, Eq. (41) reduces to Eq. (34) in the previous section (recall that $\int_{-\infty}^{\infty} d k i k \mathcal{Z}(k)=$ $\left.-2 \pi w^{\prime}(0) / c\right)$. Moreover, we can easily show that the generalized kinetic equation conserves the total number of hadrons, i.e., it satisfies Eq. (36).

Next, we seek general expressions that govern the evolution of key beam parameters, in a manner analogous to Eq. (38). Combining the definitions for the energy spread $\sigma_{h}$ and the emittance $\epsilon$ [Eq. (37)] with the new evolution equation for $F_{h}$ and neglecting the diffusion effects, we eventually obtain the expressions

$$
\begin{aligned}
\frac{d \sigma_{h}^{2}}{d t}= & -\frac{\sigma_{h}^{2}}{N_{c}^{\eta} T} \\
= & -\frac{4 r_{h} c}{\gamma T} \operatorname{Re} \int_{0}^{\infty} d k \mathcal{Z}(k) \int_{-\infty}^{\infty} d \eta \int_{0}^{\infty} d J \\
& \times \eta F_{h}\left[e^{i k Q_{0} \eta} J_{0}(k R \sqrt{J})-1\right]
\end{aligned}
$$

and

$$
\begin{aligned}
\frac{d \epsilon}{d t}= & -\frac{\epsilon}{N_{c}^{\epsilon} T} \\
= & -\frac{4 r_{h} c}{\gamma T} \operatorname{Re} \int_{0}^{\infty} d k \mathcal{Z}(k) \int_{-\infty}^{\infty} d \eta \int_{0}^{\infty} d J \\
& \times \frac{i S}{R} F_{h} \sqrt{J} J_{1}(k R \sqrt{J}) e^{i k Q_{0} \eta},
\end{aligned}
$$

where we have performed some integration by parts and also used the general property $\mathcal{Z}(-k)=\mathcal{Z}^{*}(k)$. Here, we have also reintroduced the scaled cooling times $N_{c}^{\eta}$ and $N_{c}^{\epsilon}$, first discussed in the previous section.

At this point, we find it useful to clarify a subtlety that could be potentially confusing. Since our aim is to describe the dynamics of the cooling process, the average hadron beam distribution function $F_{h}$, apart from $\eta$ and $J$, also depends on the time $t$. As a result, beam properties that are expressible as moments of $F_{h}$ (like the energy spread and emittance) are also functions of $t$. However, the reader will note that we have suppressed this time dependence in the derivation of the RHS of the kinetic equation [Eqs. (34) and (41)]. This has been done for reasons of notation simplification but it should be borne in mind that these equations are, in fact, valid for all $t$. Of course, the temporal evolution described by them is slow in the sense that its characteristic timescale is much larger than the revolution period $T$.

For the limiting case of small dispersion and chicane strength, we have seen that the cooling effect follows a simple exponential decay law [expressed by Eq. (38) if the diffusion terms are neglected]. For this example, the scaled cooling times $N_{c}^{\eta}, N_{c}^{\epsilon}$ are constant. For the more general evolution laws given by Eq. (43)-(44), the decay pattern of the beam properties is not exactly exponential and cannot be determined analytically. Moreover, the cooling times (being related to the logarithmic derivatives of the energy spread and emittance) are now functions of time. In this case, we are typically more concerned with the values of the cooling times at $t=0$. This allows us to use the initial value of the distribution function $F_{h}$ in the RHS of Eqs. (43) and (44), which is quite useful since the function $F_{h}(\eta, J, t=0)$ can be expressed analytically. Apart from facilitating our further analysis, this approximation has some practical applications as well. For some scenarios, the cooling is only meant to counteract effects such as intrabeam scattering (IBS), which would tend to reduce the brightness of the hadron beam in a collider. In such a case, we are primarily interested in matching the initial cooling times to their IBS counterparts. Having such a setup in mind, in the following section, we seek to derive expressions for the initial cooling times for the purpose of optimizing them with respect to the lattice and beam parameters.

\section{OPTIMIZATION OF THE COOLING RATES}

To start with, we introduce some simplifications regarding the MBEC configuration under consideration. In particular, we assume that $\beta_{1}=\beta_{2}=\beta_{0}, D_{1}=D_{2}=D_{0}$, $\alpha_{1}=\alpha_{2}=0$ and $D_{1}^{\prime}=D_{2}^{\prime}=0$. In this case we have $R_{33}=R_{44}=\cos \mu, R_{34}=\beta_{0} \sin \mu, R_{43}=-\sin \mu / \beta_{0}, R_{36}=$ $-(1+\cos \mu) D_{0}$, and $R_{46}=D_{0} \sin \mu / \beta_{0}$. Moreover, $S=$ $D_{0}^{2} \sin \mu / \beta_{0}$ and $R^{2}=8 D_{0}^{2} \sin ^{2}(\mu / 2) / \beta_{0}$. For the initial hadron distribution function, we select

$F_{h}=F_{h}(\eta, J, t=0)=\frac{1}{(2 \pi)^{3 / 2} \sigma_{h} \epsilon} \exp \left(-\frac{\eta^{2}}{2 \sigma_{h}^{2}}\right) \exp \left(-\frac{J}{\epsilon}\right)$,

where $\epsilon$ is the transverse (vertical) emittance and $\sigma_{h}$ is the energy spread. The fact that $\beta$ and $D$ have the same values at the modulator and the kicker ensures that the vertical size $\Sigma_{y}$ of the hadron beam is the same at these two locations. The latter quantity is expressed by $\Sigma_{y}=\left(\Sigma_{0}^{2}+D_{0}^{2} \sigma_{h}^{2}\right)^{1 / 2}$, where $\Sigma_{0}=\sqrt{\epsilon \beta_{0}}$ is the vertical size in the absence of dispersion. We also assume that the same is true of the horizontal beam size $\Sigma_{x} \equiv \Sigma$, which does not have a dispersive contribution. Thus, in our model, the hadron beam has a common (in general, elliptical) cross section in the two cooling modules where it interacts with the electron beam. The size aspect ratio of the hadron beam is $r=\Sigma_{y} / \Sigma=\sqrt{r_{0}^{2}+D_{0}^{2} \sigma_{h}^{2} / \Sigma^{2}}$, where $r_{0}=\Sigma_{0} / \Sigma$ is the aspect ratio for zero dispersion. To further simplify our treatment, we also assume that the cooler electron beam has an elliptical cross section that perfectly overlaps with that of the hadron beam at the modulator and the kicker.

Next, we introduce the following scaled quantities: $\hat{k}=k \Sigma / \gamma, q_{h}=R_{56}^{(h)} \sigma_{h} \gamma / \Sigma, q_{s}=S \sigma_{h} \gamma / \Sigma, \hat{Q}_{0}=q_{h}-q_{s}$, $q_{r}=\gamma R \sqrt{\epsilon} / \Sigma, \hat{\eta}=\eta / \sigma_{h}$, and $\hat{J}=J / \epsilon$. In terms of this scaling, the relations for the cooling times become 


$$
\begin{aligned}
\frac{1}{N_{c}^{\eta}}= & \frac{4 \epsilon r_{h} c}{\Sigma} \operatorname{Re} \int_{0}^{\infty} d \hat{k} \mathcal{Z}(\hat{k}) \int_{-\infty}^{\infty} d \hat{\eta} \int_{0}^{\infty} d \hat{J} \\
& \times \hat{\eta} F_{h}\left(e^{i \hat{k} \hat{Q}_{0} \hat{\eta}} J_{0}\left(q_{r} \hat{k} \sqrt{\hat{J}}\right)-1\right)
\end{aligned}
$$

and

$$
\begin{aligned}
\frac{1}{N_{c}^{\epsilon}}= & \frac{4 \epsilon r_{h} c}{\Sigma} \operatorname{Re} \int_{0}^{\infty} d \hat{k} \mathcal{Z}(\hat{k}) \int_{-\infty}^{\infty} d \hat{\eta} \int_{0}^{\infty} d \hat{J} \\
& \times i \frac{q_{s}}{q_{r}} F_{h} \sqrt{\hat{J}} J_{1}\left(q_{r} \hat{k} \sqrt{\hat{J}}\right) e^{i \hat{k} \hat{Q}_{0} \hat{\eta}} .
\end{aligned}
$$

The analytical expression for the impedance $\mathcal{Z}$ when no plasma amplification stage is present between the modulator and the kicker is given by

$$
\mathcal{Z}(\hat{k})=-\frac{4 i I_{e} L_{m} L_{k}}{c \Sigma^{2} \gamma^{3} I_{A} \sigma_{e}} q_{e} \hat{k} \exp \left(-\hat{k}^{2} q_{e}^{2} / 2\right) H^{2}(\hat{k}, r),
$$

where $I_{e}$ is the electron beam current, $I_{A}=e c / r_{e} \approx 17 \mathrm{kA}$ is the Alfven current $\left(r_{e}=e^{2} / m_{e} c^{2}\right.$ is the classical electron radius), $L_{m}, L_{k}$ are the lengths of the modulator and kicker sections (respectively), $\sigma_{e}$ is the rms energy spread of the electron beam and $q_{e}=R_{56}^{(e)} \sigma_{e} \gamma / \Sigma$ is the scaled electron chicane strength. Moreover, the function $H$ is given by

$H(\hat{k}, r)=\frac{i}{2} \int_{-\infty}^{\infty} d \hat{z} \Phi(\hat{z}, r) e^{-i \hat{k} \hat{z}}=\int_{0}^{\infty} d \hat{z} \Phi(\hat{z}, r) \sin (\hat{k} \hat{z})$,

where $\Phi(\hat{z}, r)$ is the space charge interaction function. The physical interpretation of $\Phi$ can be stated as follows: to describe the space charge interaction between the hadron and electron beams, we subdivide each of them into slices with charge $Z e$ and $-e$ (respectively) and a common elliptical cross section with transverse rms sizes $\Sigma_{x}=\Sigma$ and $\Sigma_{y}$. The force between two slices separated by a distance $z$ is then given by

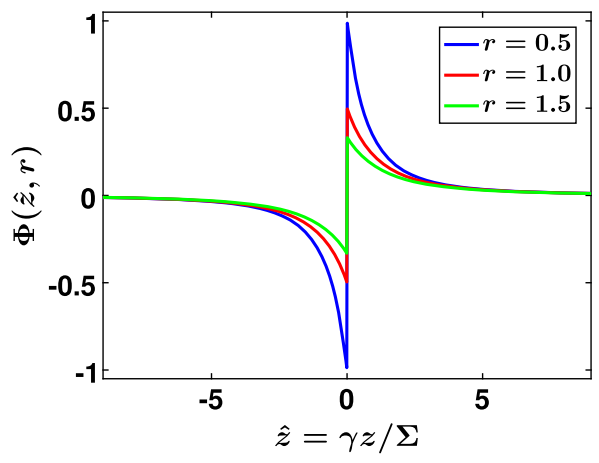

$$
F_{z}=-\frac{Z e^{2}}{\Sigma^{2}} \Phi\left(\frac{\gamma z}{\Sigma}, r\right)
$$

The function $\Phi(\hat{z}, r)$ is studied extensively in Appendix C, where a fitting formula for it is given. In Fig. 2 we plot this function for several values of the beam aspect ratio $r$. Also included is a companion plot of the function $H(\hat{k}, r)$. We note that, compared to our earlier calculation of $\mathcal{Z}$ in Ref. [5], switching to an elliptical cross section for the two beams only affects the interaction function $\Phi$, leaving the other details of our derivation unchanged.

Combining Eq. (48) with Eqs. (46) and (47), we obtain

$$
\begin{aligned}
& 1 / N_{c}^{\eta}=\frac{4 I_{e} L_{m} L_{k} r_{h}}{\Sigma^{3} \pi \gamma^{3} I_{A} \sigma_{e} \sigma_{h}} I_{\eta}, \\
& 1 / N_{c}^{\epsilon}=\frac{4 I_{e} L_{m} L_{k} r_{h}}{\Sigma^{3} \pi \gamma^{3} I_{A} \sigma_{e} \sigma_{h}} I_{\epsilon}
\end{aligned}
$$

and

$I_{\eta}=q_{e} \operatorname{Re} \int_{0}^{\infty} d \hat{k} \hat{k} \exp \left(-\hat{k}^{2} q_{e}^{2} / 2\right) H^{2}(\hat{k}, r) R_{\eta}(\hat{k})$,

$I_{\epsilon}=q_{e} \operatorname{Re} \int_{0}^{\infty} d \hat{k} \hat{k} \exp \left(-\hat{k}^{2} q_{e}^{2} / 2\right) H^{2}(\hat{k}, r) R_{\epsilon}(\hat{k})$,

where

$R_{\eta}(\hat{k})=4 \pi i \sigma_{h} \epsilon \int_{-\infty}^{\infty} d \hat{\eta} \hat{\eta} \int_{0}^{\infty} d \hat{J} F_{h}\left(1-e^{i \hat{k} \hat{Q}_{0} \hat{\eta}} J_{0}\left(q_{r} \hat{k} \sqrt{\hat{J}}\right)\right)$

and

$R_{\epsilon}(\hat{k})=4 \pi \sigma_{h} \epsilon \frac{q_{s}}{q_{r}} \int_{-\infty}^{\infty} d \hat{\eta} \int_{0}^{\infty} d \hat{J} F_{h} \sqrt{\hat{J}} e^{i \hat{k} \hat{Q}_{0} \hat{\eta}} J_{1}\left(q_{r} \hat{k} \sqrt{\hat{J}}\right)$.

Substituting the initial beam distribution of Eq. (45), which can be written as

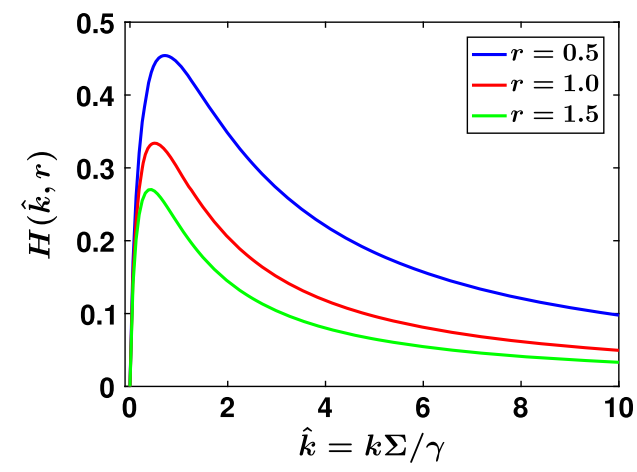

FIG. 2. Left panel: Plot of the interaction function $\Phi(\hat{z}, r)$ in terms of $\hat{z}$, for three different values of the aspect ratio $r$. Right panel: Plot of the function $H(\hat{k}, r)$ in terms of $\hat{k}$, for the same values of $r$. 


$$
F_{h}=\frac{1}{(2 \pi)^{3 / 2} \sigma_{h} \epsilon} \exp \left(-\frac{\hat{\eta}^{2}}{2}\right) \exp (-\hat{J}),
$$

into Eqs. (53) and (54), we find that

$$
R_{\eta}(\hat{k})=2 \hat{Q}_{0} \hat{k} \exp \left(-\frac{\hat{k}^{2} \hat{Q}_{0}^{2}}{2}-\frac{\hat{k}^{2} q_{r}^{2}}{4}\right)
$$

and

$$
R_{\epsilon}(\hat{k})=q_{s} \hat{k} \exp \left(-\frac{\hat{k}^{2} \hat{Q}_{0}^{2}}{2}-\frac{\hat{k}^{2} q_{r}^{2}}{4}\right) .
$$

As a consequence, the integrals $I_{\eta}$ and $I_{\epsilon}$ become

$$
\begin{aligned}
I_{\eta}= & 2\left(q_{h}-q_{s}\right) q_{e} \int_{0}^{\infty} d \hat{k} \hat{k}^{2} \exp \left[-\hat{k}^{2}\left(q_{e}^{2}+\left(q_{h}-q_{s}\right)^{2}\right) / 2\right. \\
& \left.-\hat{k}^{2} q_{r}^{2} / 4\right] H^{2}(\hat{k}, r)
\end{aligned}
$$

and

$$
\begin{aligned}
I_{\epsilon}= & q_{s} q_{e} \int_{0}^{\infty} d \hat{k} \hat{k}^{2} \exp \left[-\hat{k}^{2}\left(q_{e}^{2}+\left(q_{h}-q_{s}\right)^{2}\right) / 2-\hat{k}^{2} q_{r}^{2} / 4\right] \\
& \times H^{2}(\hat{k}, r),
\end{aligned}
$$

where we recall that $\hat{Q}_{0}=q_{h}-q_{s}$. From the above results, we can readily deduce a simple relationship between the cooling times for the energy spread and the transverse emittance, namely

$$
\frac{N_{c}^{\epsilon}}{N_{c}^{\eta}}=\frac{2 \hat{Q}_{0}}{q_{s}}=2 \frac{q_{h}-q_{s}}{q_{s}} .
$$

This result for the ratio of the cooling times is identical to the one derived (in a different context) at the end of Sec. IV.

From an inspection of Eqs. (58) and (59), we obtain some important preliminary insight regarding the influence of dispersion on the cooling effect. To begin with, for zero dispersion $\left(D_{0}=0\right) R^{2}=S=0$ so $q_{r}=q_{s}=0$. In this case, $I_{\epsilon}=0$ and there is no emittance variation (cooling or heating). Moreover, the expression for the longitudinal cooling rate [Eq. (58)] reduces to the one obtained in our earlier 1D analysis-in Ref. [5]. For nonzero dispersion one can have cooling of both the energy spread and the transverse emittance, provided that $q_{h}>q_{s}$. An interesting situation occurs when the dispersion and the hadron chicane strength are such that $q_{h}=q_{s}$ (or $\hat{Q}_{0}=0$ ), in which case the longitudinal rate becomes zero $\left(I_{\eta}=0\right)$. For $q_{h}<q_{s}$, one still has emittance cooling but now this is accompanied by heating in terms of the energy spread.

Even though the expressions for the cooling integrals are more complicated than their 1D counterparts, they still allow us to optimize the parameters of a relatively simple MBEC configuration. To demonstrate this, let us consider a scenario in which all parameters are fixed except $D_{0}, \mu$, $R_{56}^{(h)}$, and $R_{56}^{(e)}$. Moreover, we choose to confine our attention to the case of zero cooling for the energy spread. As we have seen, this simplification removes one d.o.f. since $R_{56}^{(h)}$ is always chosen so that $q_{h}=q_{s}$. Our objective is to maximize the emittance cooling rate $1 / N_{c}^{\epsilon}$, which is equivalent to optimizing $I_{\epsilon}$. After some manipulation, Eq. (59) can be rewritten as

$$
\begin{aligned}
I_{\epsilon}= & q_{e} \sqrt{u v^{2}-u^{2} /\left(4 \Upsilon^{2}\right)} \int_{0}^{\infty} d \hat{k} \hat{k}^{2} H^{2}\left(\hat{k}, r_{0} \sqrt{1+v^{2}}\right) \\
& \times \exp \left[-\left(q_{e}^{2}+u\right) \frac{\hat{k}^{2}}{2}\right] .
\end{aligned}
$$

Here, we have expressed the beam aspect ratio $r$ as $r=$ $r_{0} \sqrt{1+v^{2}}$, where $v \equiv \sigma_{h} D_{0} / \Sigma_{0}$ and we have also introduced the variables $u=q_{r}^{2} / 2$ and $\Upsilon=\gamma \epsilon /\left(\Sigma \sigma_{h}\right)$. The main advantage of this rearrangement is that the effect of dispersion is now contained in only two parameters $\left(u\right.$ and $v$ ). Expressed in this form, $I_{\epsilon}$ can be maximized using a typical unconstrained optimization software package. For a numerical illustration, we have considered a parameter set that is representative of the proposed eRHIC electron-ion collider (see Table I). Assuming $\beta_{x}=\beta_{y}=$ $\beta_{0} \approx 50 \mathrm{~m}$, we have $\Sigma=\sqrt{\epsilon_{x} \beta_{x}} \approx 680 \mu \mathrm{m}$ and $\Sigma_{0}=$ $\sqrt{\epsilon_{y} \beta_{y}}=\sqrt{\epsilon \beta_{0}} \approx 250 \mu \mathrm{m}$. Thus the aspect ratio without dispersion is $r_{0}=\Sigma_{0} / \Sigma=0.37$ while $\Upsilon=\gamma \epsilon /\left(\Sigma \sigma_{h}\right)=$ 1.24. For these parameters the optimum value of $I_{\epsilon}$ is 0.103 , obtained for $u=0.229, v=1.581$, and $q_{e}=0.482$ (we also have $q_{s}=0.75$ ). In terms of the original (dimensional) quantities, this optimum point yields a dispersion $D_{0}=\beta_{0}(\Upsilon v) /\left(\gamma r_{0}\right) \approx 0.87 \mathrm{~m}$ and a phase advance $\mu=$ $2 \sin ^{-1}(\sqrt{u} /(2 \Upsilon v)) \approx 0.24$. The hadron/electron chicane strengths are $R_{56}^{(h)}=q_{h} \Sigma /\left(\gamma \sigma_{h}\right)=3.8 \mathrm{~mm}$ and $R_{56}^{(e)}=$ $q_{e} \Sigma /\left(\gamma \sigma_{e}\right)=1.1 \mathrm{~cm}$.

In order to verify that the above-mentioned parameters correspond to an optimum solution, we scan the dispersion while keeping all other quantities fixed and plot the cooling

TABLE I. Parameters of the eRHIC collider with a hypothetical MBEC cooling section.

\begin{tabular}{lc}
\hline \hline Proton energy $\gamma m_{h} c^{2}[\mathrm{GeV}]$ & 275 \\
Proton relative energy spread, $\sigma_{h}$ & $4.6 \times 10^{-4}$ \\
Electron energy $\gamma m_{e} c^{2}[\mathrm{MeV}]$ & 150 \\
Electron relative energy spread, $\sigma_{e}$ & $1 \times 10^{-4}$ \\
Relativistic factor, $\gamma$ & 293 \\
Electron beam current, $I_{e}[\mathrm{~A}]$ & 30 \\
Electron bunch charge, $Q_{e}[\mathrm{nC}]$ & 1 \\
Proton rms bunch length, $\sigma_{z}^{(h)}[\mathrm{cm}]$ & 5 \\
Revolution period $T[\mathrm{~s}]$ & $1.2 \times 10^{-5}$ \\
Horizontal/vertical proton emittance $\epsilon_{x} / \epsilon_{y}[\mathrm{~nm}]$ & $9.2 / 1.3$ \\
Modulator and kicker lengths $L_{m}, L_{k}[\mathrm{~m}]$ & 50 \\
\hline \hline
\end{tabular}


times as a function of $D_{0}$. From Eq. (51), we recall that the scaled cooling times are given by $1 / N_{c}^{\eta}=A_{0} I_{\eta}$ and $1 / N_{c}^{\epsilon}=A_{0} I_{\epsilon}$, where the dimensionless prefactor $A_{0}$ is given by

$$
A_{0}=\frac{4 I_{e}^{\mathrm{eff}} L_{m} L_{k} r_{h}}{\Sigma^{3} \pi \gamma^{3} I_{A} \sigma_{e} \sigma_{h}}
$$

Here, the peak electron beam current $I_{e}$ has been replaced by an effective value of $I_{e}^{\text {eff }} \approx c Q_{e} /\left(\sqrt{2 \pi} \sigma_{z}^{(h)}\right) \approx 2.4 \mathrm{~A}$. This is necessary in order to account for the finite longitudinal size of both beams, which are assumed to have Gaussian current profiles (more details can be found in Ref. [6]). The results of the dispersion scan are given in Fig. 3. A minimum of the transverse cooling time is indeed observed at $D_{0}=D_{\text {opt }}=0.87 \mathrm{~m}$, the minimum value being $\sim 18 \mathrm{~h}$. As we ramp up the dispersion, we observe a transition from a state of zero transverse cooling (for $D_{0}=0$ ) to a state of zero longitudinal cooling (for $D_{0}=D_{\text {opt }}$ ), followed by heating of the energy spread and suboptimal cooling of the emittance. We also note that the case with $D_{0}=0$ corresponds to optimum longitudinal cooling (with a timescale $\approx 7.7 \mathrm{~h}$ ). Finally, for $D_{0} \approx 0.82 D_{\mathrm{opt}} \approx 0.72 \mathrm{~m}$, we have $t_{c}^{(\eta)} \approx t_{c}^{(\epsilon)} \approx 22 \mathrm{~h}$.

\section{COMPARISON WITH SIMULATION}

In order to benchmark our analytical theory, we have carried out simulations of MBEC that take into account the transverse motion of the hadrons. In these simulations, electrons and hadrons are represented by macroparticles (slices) that interact with the force given by Eq. (50). Initially, $N_{e}$ electron macroparticles are randomly distributed in the interval $0<z<\Delta z$ with the energy $\eta_{i}^{(e)}$ of the $i$ th electron randomly assigned from a Gaussian distribution with an rms width $\sigma_{e}$. Periodic boundary conditions are imposed at the boundaries of the interval $[0, \Delta z]$. As far as the hadron coordinates are concerned, the energy $\eta^{(h)}$,

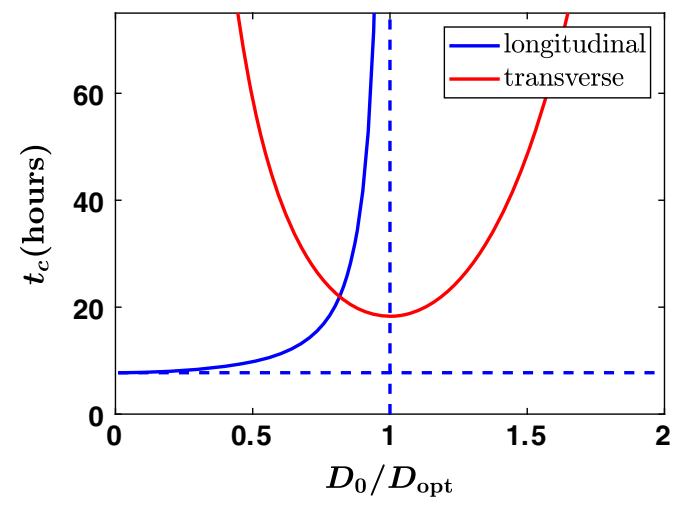

FIG. 3. Transverse and longitudinal cooling times as a function of dispersion. vertical slope $P=d y / d s$ and betatron position $\bar{y}$ are all drawn from Gaussian distributions with widths $\sigma_{h}, \Sigma_{0}{ }^{\prime}=$ $\sqrt{\epsilon / \beta_{0}}$ and $\Sigma_{0}=\sqrt{\epsilon \beta_{0}}$ (respectively). In the presence of dispersion the hadron vertical position is $y=D_{0} \eta^{(h)}+\bar{y}$. Such a hadron macroparticle is placed at a random location within the interval and the energy of each electron $i$ is changed by $\Delta \eta_{i}^{(e)}=f_{z, i} L_{m} / \gamma m_{e} c^{2}$, where $f_{z, i}$ is the force exerted by the hadron on electron $i$. On the next step, corresponding to the passage of the hadrons from the modulator to the kicker, the hadron coordinate vector $\left(z, \eta^{(h)}, y, P\right)^{T}$ is transformed according to the transport matrix $R_{t}$, which is given by Eq. (14). On the other hand, the electrons pass through the chicane $R_{56}^{(e)}$ where they are shifted longitudinally by $R_{56}^{(e)}\left(\eta_{i}^{(e)}+\Delta \eta_{i}^{(e)}\right)$. Finally, in the kicker itself, the hadron energy is changed from $\eta^{(h)}$ to $\eta^{(h)}+\Delta \eta^{(h)}$ with $\Delta \eta^{(h)}=\sum_{i=1}^{N_{e}} f_{z, i} L_{k} / \gamma m_{h} c^{2}$, where now $f_{z, i}$ denotes the force acting on the hadron from $i$ th electron. Because of the dispersion $D_{0}$, this energy kick also results in a variation of the action variable $J$. After the kick, the action change is calculated with the help of Eq. (1), $\Delta J=J_{\text {final }}-J_{\text {init }}$. This procedure is repeated $M$ times and the longitudinal cooling rate is estimated as an average over $M$ runs of the difference $\left(\eta^{(h)}+\Delta \eta^{(h)}\right)^{2}-\sigma_{h}^{2}$. An entirely analogous procedure for $\Delta J$ yields the transverse cooling rate.

By a proper scaling of the dimensional variables of the simulation problem, one can show that it is determined by several dimensionless parameters. The first one, $\nu=n_{0 e} \Sigma / \gamma$, is equal to the number of electrons within a length $\Sigma / \gamma$, a quantity that is proportional to the electron beam current $I_{e}$. Two more parameters, $A_{1}$ and $A_{2}$, characterize the interaction strength in the modulator and the kicker normalized by the electron and hadron energy spread, respectively,

$$
A_{1}=\frac{Z r_{e} L_{m}}{\gamma \Sigma^{2} \sigma_{e}}, \quad A_{2}=\frac{r_{h} L_{k}}{Z \gamma \Sigma^{2} \sigma_{h}} .
$$

Furthermore, apart from the dimensionless chicane strengths of $q_{e}$ and $q_{h}$, consideration of the transverse motion introduces some additional scaled parameters into the simulation setup. Almost all of these, namely the phase advance $\mu$, the zero-dispersion aspect ratio $r_{0}$ of the hadron beam and $v=\sigma_{h} D_{0} / \Sigma_{0}$, have already been mentioned in the previous sections (the only new addition being the variable $v_{1} \equiv \gamma D_{0} / \beta_{0}$ ). Calculating the numerical values of $\nu, A_{1}$ and $A_{2}$ for the eRHIC parameters in Table I, we find

$\nu=1.5 \times 10^{6}, \quad A_{1}=7.8 \times 10^{-6}, \quad A_{2}=9.3 \times 10^{-10}$.

Simulations with these values are computationally challenging due to the large number of required macroparticles and small values of the interaction strengths. To avoid this 


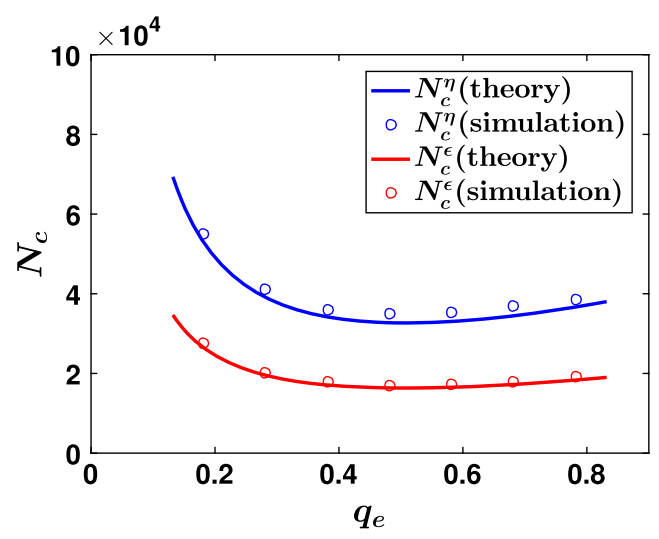

FIG. 4. Simulated vs theoretical cooling times.

problem we used larger values for $A_{1}$ and $A_{2}$ and smaller values for $\nu$, in particular

$\nu=5 \times 10^{2}, \quad A_{1}=2 \times 10^{-2}, \quad A_{2}=5 \times 10^{-5}$.

For the other scaled parameters, we use the dispersion and phase advance values obtained from the optimization study of the previous section. These yield $\mu=0.24, r_{0}=0.37$, $v=1.58$ and $v_{1}=5.25$. As far as the chicane strengths are concerned, we scan $q_{e}$ around its optimum value of 0.482 for a fixed $q_{h}=1.25 q_{s}=0.94$. The results of this scan are shown in Fig. 4, where we plot the scaled cooling times as a function of $q_{e}$. Good agreement is observed between theory and simulation for both the transverse and the longitudinal cooling time. A deviation of a few percent can probably be attributed to diffusion effects, which can also be tracked analytically. Of course, our simulations have a limited scope in the sense that they do not represent the output of a fully 3D algorithm. Some preliminary work regarding a 3D MBEC simulation (and theory) is presented in Ref. [8].

\section{ADDITION OF PLASMA AMPLIFICATION STAGES}

The analysis carried out so far refers to an MBEC configuration with no plasma amplification stages. Each of the latter typically consists of a long drift section (the length being comparable to the plasma oscillation wavelength of the electron beam) followed by a chicane. Including any number of such stages after the first electron chicane would only change the expression for the impedance, which would be modified according to $\mathcal{Z}(\hat{k}) \rightarrow \mathcal{Z}(\hat{k}) G(\hat{k})$.
Here, $G(\hat{k})$ is a gain factor that depends on the various parameters of the plasma stages (drift lengths, added chicane strengths, local electron beam size etc). Analytical expressions for $G$ are available in [6] for some simple cascade configurations. Incorporating this new space charge impedance in our formalism is a straightforward exercise; it merely adds a multiplicative factor $\propto G$ to the integrand of $I_{\eta}$ and $I_{\epsilon}$, while also boosting the value of the prefactor $A_{0}$ by a corresponding constant. Specifically, for a cascade of two plasma stages (as shown in Fig. 5), the transverse cooling integral becomes

$$
\begin{aligned}
I_{\epsilon}^{\prime}= & q_{s} q_{e, 1} \int_{0}^{\infty} d \hat{k} \hat{k}^{2} \exp \left\{-\hat{k}^{2}\left[q_{e, 1}^{2}\right.\right. \\
& \left.\left.+\left(q_{h}-q_{s}\right)^{2}\right] / 2-\hat{k}^{2} q_{r}^{2} / 4\right\} \\
& \times H^{2}(\hat{k}, r) g_{1}(\hat{k}) g_{2}(\hat{k}),
\end{aligned}
$$

with

$$
\begin{aligned}
g_{1}(\hat{k})= & \sqrt{\frac{\hat{k} H_{1}\left(r_{p} \hat{k}\right)}{r_{p}}} q_{e, 2} \exp \left(-\hat{k}^{2} q_{e, 2}^{2} / 2\right) \\
& \times \sin \left(r_{p} \frac{\Omega_{p} L_{d}}{c} \sqrt{\frac{2 \hat{k} H_{1}\left(r_{p} \hat{k}\right)}{r_{p}}}\right)
\end{aligned}
$$

and

$$
\begin{aligned}
g_{2}(\hat{k})= & \sqrt{\frac{\hat{k} H_{1}\left(r_{p} \hat{k}\right)}{r_{p}}} q_{e, 3} \exp \left(-\hat{k}^{2} q_{e, 3}^{2} / 2\right) \\
& \times \sin \left(r_{p} \frac{\Omega_{p} L_{d}}{c} \sqrt{\frac{2 \hat{k} H_{1}\left(r_{p} \hat{k}\right)}{r_{p}}}\right),
\end{aligned}
$$

being the contributions of the individual plasma stages. Moreover, $H_{1}(\hat{k}) \equiv H(\hat{k}, r=1), q_{e, j}=R_{56}^{(e, j)} \sigma_{e} \gamma / \Sigma$ (for $j=1,2,3)$ are the scaled electron chicane strengths, $L_{d}$ is the common length of the two drift sections and $\Omega_{p}=$ $\left(c / r_{p} \Sigma\right)\left(I_{e} / \gamma^{3} I_{A}\right)^{1 / 2}$ is the plasma oscillation frequency. Here, $r_{p} \Sigma \equiv \Sigma_{p}$ represents the local electron beam size at the plasma stages, the beam being assumed round at these two locations. The emittance cooling time is now given by $1 / N_{c}^{\epsilon}=A_{0}^{\prime} I_{\epsilon}^{\prime}$, where the new prefactor is expressed by

$$
A_{0}^{\prime}=\frac{8\left(\bar{I}_{e}^{\mathrm{fff}}\right)^{2} L_{m} L_{k} r_{h}}{\Sigma^{3} \pi \gamma^{4} I_{A}^{2} \sigma_{e}^{3} \sigma_{h}}
$$

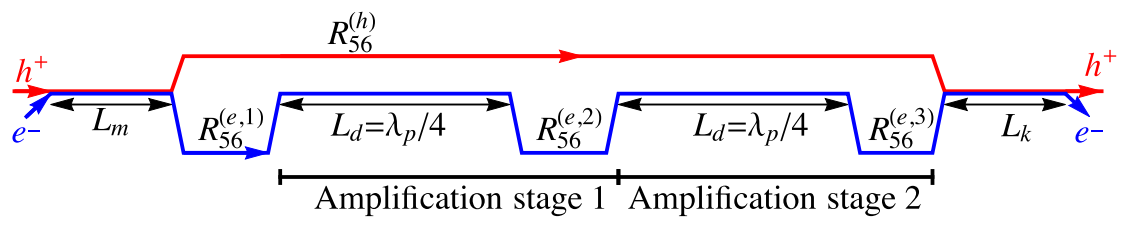

FIG. 5. An MBEC configuration with two plasma amplification stages. 
Here, $\bar{I}_{\mathrm{eff}}=2^{-1 / 4} I_{e} \sqrt{\sigma_{z}^{(e)} / \sigma_{z}^{(h)}}$ is the effective electron beam current for the case of two plasma stages and $\sigma_{z}^{(e)}=$ $c Q_{e} /\left(\sqrt{2 \pi} I_{e}\right)$ is the electron rms bunch length. The cooling time for the energy spread can still be expressed by Eq. (60).

Using these updated expressions a lattice parameter optimization is possible, in a manner entirely analogous to the case with no plasma stages. For the eRHIC parameter set of Table I, we assume that the e-beam at the plasma stages can be squeezed to a size $r_{p} \Sigma \approx 135 \mu \mathrm{m}$ (so that $\left.r_{p}=0.2\right)$. The optimum cooling time $\left[\sim\left(A_{0}^{\prime} I_{\epsilon}^{\prime}\right)^{-1}\right]$ is now about $4 \mathrm{~min}$. As far as the optimized machine parameters are concerned, we have $D_{0} \approx 1.3 \mathrm{~m}, \mu \approx 0.37$, $R_{56}^{(h)}=1.25 \mathrm{~cm}, \quad R_{56}^{(e, 1)}=R_{56}^{(e, 2)}=R_{56}^{(e, 3)}=2.5 \mathrm{~cm}$. The necessary drift length is $L_{d} \approx \Sigma \gamma^{3 / 2} \sqrt{I_{A} / I_{e}} \approx 81 \mathrm{~m}$. Thus we see that, by adding amplification stages, the cooling time can be greatly reduced, though diffusion effects and nonlinear behavior in the plasma modules may also become more important than before [6].

\section{SUMMARY}

In this paper, we have developed a theoretical formalism that describes the influence of transverse beam dynamics on the mechanism of microbunched electron cooling (MBEC). Extending our earlier one-dimensional (1D) treatment, we modify our kinetic theory-based approach in order to include the transverse (betatron) motion of the hadron beam. The latter is treated in a systematic and rigorous fashion that explicitly takes into account the interplay between the transverse and longitudinal d.o.f. This allows us to quantify the effect of the various hadron lattice functions on the cooling of the energy spread. More importantly, it also enables us to demonstrate how some of these parameters - in particular, the dispersion-are responsible for the cooling of the transverse emittance. The crucial role of dispersion in generating emittance cooling has also been emphasized in the context of optical stochastic cooling [9]. To ensure that our analysis does not become prohibitively cumbersome, we only consider the vertical component of the hadron betatron motion and keep the other aspects of our derivation in a purely 1D framework. In particular, the space charge interaction is described in terms of a 1D Gaussian slice model while the transverse motion of the cooler electron beam is completely neglected. These assumptions do impose some restrictions on the applicability of our model; in particular, we cannot use it for a fully rigorous study of how the various details of a realistic beamline would affect MBEC. However, our work does result in relatively simple analytical formulas for the transverse and longitudinal cooling times, which are subsequently verified through comparison with simulation. We have used these expressions in an optimization study of a conceptual MBEC configuration for the eRHIC collider. The basic conclusion is that, by a proper choice of the hadron lattice properties (i.e., dispersion, phase advance etc), one can obtain a similar degree of cooling for both the emittance and the energy spread. The resulting requirements for the lattice are not excessive (the necessary dispersion value being about $1 \mathrm{~m}$, the chicane strength $\sim 1 \mathrm{~cm}$ ) but the obtained cooling times are rather high $(\sim 20 \mathrm{~h})$. This can be improved through the use of plasma amplification stages, which aim to enhance the bunching of the electron beam and reduce the cooling times to levels that allow us to counteract effects such as intrabeam scattering ( $\sim 1 \mathrm{~h}$ or lower). Our analysis can easily incorporate this important feature, and parameter optimization is still feasible in this case. The addition of two plasma stages, each with a length $\sim 80 \mathrm{~m}$, appears to be sufficient for our purposes. Overall, this work effectively complements Refs. [5,6] by showing that MBEC can provide transverse, as well as longitudinal, cooling for a hadron beam. We expect that the methods outlined in this paper will be a useful addition to the toolbox required for future studies of MBEC.

\section{ACKNOWLEDGMENTS}

We would like to thank F. Willeke and A. Zholents for useful discussions and comments regarding the subject of this paper. This work was supported by the Department of Energy, Contract No. DE-AC02-76SF00515.

\section{APPENDIX A: KINETIC EQUATION IN THE LIMIT OF SMALL DISPERSION AND HADRO CHICANE STRENGTH}

The first four terms of Eq. (33) (i.e., those up to the second line of the said equation) are responsible for the diffusion effects. In order to calculate the averages involved in them, we use Eqs. (24) and (26) in conjunction with Eqs. (12) and (28). Moreover, we have the following complication: since the cooling is a slow process that takes place over many revolutions around the ring, a particular hadron each time arrives at the cooler with approximately the same values of $J$ and $\eta$, but different betatron phases $\phi$. Thus, apart from ensemble averaging, we also need to average $\Delta f$ over the betatron angle $\phi$. We denote the averaging over the phase $\phi$ by the symbols $\triangleleft \ldots \triangleright$. It is straightforward to show that $\triangleleft\left\langle\Delta \eta^{(h)} \Delta \overline{\boldsymbol{J}}^{(h, 1)}\right\rangle \triangleright=0$ and

$$
\begin{aligned}
\triangleleft\left\langle\left(\Delta \overline{\boldsymbol{J}}^{(h, 1)}\right)^{2}\right\rangle \triangleright & =-2 J\left\langle\Delta \overline{\boldsymbol{J}}^{(h, 2)}\right\rangle \\
& =J \frac{\left\langle\left(\Delta \eta^{(h)}\right)^{2}\right\rangle}{\beta_{2}}\left[D_{2}^{2}+\left(\beta_{2} D_{2}^{\prime}+\alpha_{2} D_{2}\right)^{2}\right],
\end{aligned}
$$

in view of which the diffusion terms add up to 


$$
\begin{aligned}
& \frac{1}{2} n_{0 h}\left\langle\left(\Delta \eta^{(h)}\right)^{2}\right\rangle \partial_{\eta \eta} F_{h}+n_{0 h} \frac{\left\langle\left(\Delta \eta^{(h)}\right)^{2}\right\rangle}{2 \beta_{2}} \\
& \quad \times\left[D_{2}^{2}+\left(\beta_{2} D_{2}^{\prime}+\alpha_{2} D_{2}\right)^{2}\right]\left(\partial_{J} F_{h}+J \partial_{J J} F_{h}\right) .
\end{aligned}
$$

The average of $\left(\Delta \eta^{(h)}\right)^{2}$ is, in turn, given by

$$
\begin{aligned}
\left\langle\left(\Delta \eta^{(h)}\right)^{2}\right\rangle= & \left(\frac{r_{h} c}{2 \pi \gamma}\right)^{2} \int_{-\infty}^{\infty} d k d k^{\prime} \mathcal{Z}(k) \mathcal{Z}\left(k^{\prime}\right) \\
& \times e^{i k z+i k^{\prime} z}\left\langle\delta \hat{n}_{k^{\prime}}^{(M)} \delta \hat{n}_{k}^{(M)}\right\rangle \\
= & \frac{n_{0 h}}{2 \pi}\left(\frac{r_{h} c}{\gamma}\right)^{2} \int_{-\infty}^{\infty} d k|\mathcal{Z}(k)|^{2} .
\end{aligned}
$$

Combining the above, the diffusion terms can be written in a compact fashion as:

$$
\begin{aligned}
& \frac{n_{0 h}^{2}}{4 \pi}\left(\frac{r_{h} c}{\gamma}\right)^{2} \int_{-\infty}^{\infty} d k|\mathcal{Z}(k)|^{2} \\
& \quad \times\left[\frac{\partial^{2} F_{h}}{\partial \eta^{2}}+\frac{D_{2}^{2}+\left(\beta_{2} D_{2}^{\prime}+\alpha_{2} D_{2}\right)^{2}}{\beta_{2}} \frac{\partial}{\partial J}\left(J \frac{\partial F_{h}}{\partial J}\right)\right] .
\end{aligned}
$$

The next three terms [in the third line of Eq. (33)] are due to the self-interaction of particles. In fact, we can show that only the first of these is nonzero. To demonstrate this, we start from Eq. (13), which can be restated as

$$
\left\langle\delta \hat{n}_{k}^{(M)} \delta f^{(M)}(z, \eta, J, \phi-\mu)\right\rangle=n_{0 h} F_{h}(\eta, J) e^{-i k z}
$$

As a direct result of the above relation, we have

$$
\begin{aligned}
\left\langle\delta \hat{n}_{k}^{(M)} \partial_{\eta} \delta f^{(M)}\right\rangle & =n_{0 h} \partial_{\eta} F_{h} e^{-i k z}, \\
\left\langle\delta \hat{n}_{k}^{(M)} \partial_{J} \delta f^{(M)}\right\rangle & =n_{0 h} \partial_{J} F_{h} e^{-i k z}, \\
\left\langle\delta \hat{n}_{k}^{(M)} \partial_{\phi} \delta f^{(M)}\right\rangle & =0, \\
\left\langle\delta \hat{n}_{k}^{(M)} \partial_{z} \delta f^{(M)}\right\rangle & =-i k n_{0 h} F_{h} e^{-i k z} .
\end{aligned}
$$

Combining the first of these results with Eq. (28), we obtain

$$
\begin{aligned}
-\left\langle\Delta \eta^{(h)} \partial_{\eta} \delta f^{(M)}\right\rangle & =\frac{r_{h} c}{2 \pi \gamma} \int_{-\infty}^{\infty} d k \mathcal{Z}(k)\left\langle\delta \hat{n}_{k}^{(M)} \partial_{\eta} \delta f^{(M)}\right\rangle e^{i k z} \\
& =\frac{r_{h} c}{2 \pi \gamma} n_{0 h} \partial_{\eta} F_{h} \int_{-\infty}^{\infty} d k \mathcal{Z}(k) \\
& =-n_{0 h} \frac{r_{h}}{\gamma} w(0) \partial_{\eta} F_{h}
\end{aligned}
$$

Here, we have introduced the value of the wake function $w(z)$ at the origin $z=0$. The wake is given in terms of the impedance $\mathcal{Z}(k)$ by

$$
w(z)=-\frac{c}{2 \pi} \int_{-\infty}^{\infty} d k \mathcal{Z}(k) e^{i k z}
$$

The fact that $w(z)$ is a real quantity requires that $\mathcal{Z}(-k)=$ $\mathcal{Z}^{*}(k)$ (see Ref. [5]). The term given by Eq. (A7) does not have an impact in the cooling process and is typically ignored in the analysis even if $w(0)$ is nonzero. For the second of the self-interaction terms, Eq. (A6)_along with Eqs. (26) and (28)_-gives

$$
\begin{aligned}
-\left\langle\Delta \bar{J}^{(h, 1)} \partial_{J} \delta f^{(M)}\right\rangle \\
\rightarrow \frac{r_{h} c}{2 \pi \gamma} \sqrt{\frac{2 J}{\beta_{2}}} \triangleleft\left(\left(\beta_{2} D_{2}^{\prime}+\alpha_{2} D_{2}\right) \sin \phi-D_{2} \cos \phi\right) \triangleright \\
\quad \times \int_{-\infty}^{\infty} d k \mathcal{Z}(k)\left\langle\delta \hat{n}_{k}^{(M)} \partial_{J} \delta f^{(M)}\right\rangle e^{i k z}=0
\end{aligned}
$$

as a result of the $\phi$-averaging. An entirely analogous reasoning shows that the final term is zero, i.e.,

$$
\left\langle\Delta \phi^{(h)} \partial_{\phi} \delta f^{(M)}\right\rangle=0
$$

Next, we move on to the remaining four terms of Eq. (33), which contain the cooling effect. For these we need a corollary of Eq. (A5), namely

$$
\begin{aligned}
\left\langle\delta \hat{n}_{k}^{(M)} \partial_{z \eta} \delta f^{(M)}\right\rangle & =-i k n_{0 h} \partial_{\eta} F_{h} e^{-i k z}, \\
\left\langle\delta \hat{n}_{k}^{(M)} \partial_{z J} \delta f^{(M)}\right\rangle & =-i k n_{0 h} \partial_{J} F_{h} e^{-i k z}, \\
\left\langle\delta \hat{n}_{k}^{(M)} \partial_{z \phi} \delta f^{(M)}\right\rangle & =0 .
\end{aligned}
$$

To begin with, we calculate the term proportional to $\left\langle\Delta \eta^{(h)} \partial_{z \eta} \delta f^{(M)}\right\rangle$. We have

$$
\begin{aligned}
- & Q\left\langle\Delta \eta^{(h)} \partial_{z \eta} \delta f^{(M)}\right\rangle \\
& \rightarrow \triangleleft Q \triangleright \frac{r_{h} c}{2 \pi \gamma} \int_{-\infty}^{\infty} d k \mathcal{Z}(k) e^{i k z} \partial_{z \eta}\left\langle\delta \hat{n}_{k}^{(M)} \delta f^{(M)}\right\rangle \\
& =\frac{i r_{h} c}{2 \pi \gamma} n_{0 h} Q_{0} \eta \partial_{\eta} F_{h} \int_{-\infty}^{\infty} d k k \mathcal{Z}(k) \\
& =-w^{\prime}(0) \frac{r_{h}}{\gamma} n_{0 h} Q_{0} \eta \partial_{\eta} F_{h},
\end{aligned}
$$

where we have used Eq. (A8) to show that $i \int_{-\infty}^{\infty} d k k \mathcal{Z}(k)=$ $-2 \pi w^{\prime}(0) / c$ and Eq. (21) to obtain $\triangleleft Q \triangleright=-Q_{0} \eta$. The next (action-related) term is 


$$
\begin{aligned}
- & Q\left\langle\Delta \bar{J}^{(h, 1)} \partial_{z J} \delta f^{(M)}\right\rangle \\
& \rightarrow \hat{Q}_{J} \frac{r_{h} c}{2 \pi \gamma} \sqrt{\frac{2 J}{\beta_{2}}} \int_{-\infty}^{\infty} d k \mathcal{Z}(k) e^{i k z} \partial_{z J}\left\langle\delta \hat{n}_{k}^{(M)} \delta f^{(M)}\right\rangle \\
& =-\hat{Q}_{J} \frac{i r_{h} c}{2 \pi \gamma} n_{0 h} \sqrt{\frac{2 J}{\beta_{2}}} \partial_{J} F_{h} \int_{-\infty}^{\infty} d k k \mathcal{Z}(k) \\
& =\hat{Q}_{J} \frac{r_{h}}{\gamma} n_{0 h} w^{\prime}(0) \sqrt{\frac{2 J}{\beta_{2}}} \partial_{J} F_{h},
\end{aligned}
$$

\section{APPENDIX B: KINETIC EQUATION-GENERAL CASE}

By revisiting Eq. (27), we see that, in general, the cooling and self-interaction terms are contained in the sum

$$
\begin{aligned}
\left\langle\partial_{z} \delta f^{(M)}\left(z-Q_{0} \eta+\bar{Q}, \eta, J, \phi-\mu\right) R_{56}^{(h)} \Delta \eta^{(h)}\right\rangle & -\left\langle\partial_{2} \delta f^{(M)}\left(z-Q_{0} \eta+\bar{Q}, \eta, J, \phi-\mu\right) \Delta \eta^{(h)}\right\rangle \\
- & \left\langle\partial_{3} \delta f^{(M)}\left(z-Q_{0} \eta+\bar{Q}, \eta, J, \phi-\mu\right) \Delta \bar{J}^{(h, 1)}\right\rangle \\
& -\left\langle\partial_{\phi} \delta f^{(M)}\left(z-Q_{0} \eta+\bar{Q}, \eta, J, \phi-\mu\right) \Delta \phi^{(h)}\right\rangle,
\end{aligned}
$$

where

$$
\begin{aligned}
\hat{Q}_{J} & =\triangleleft Q\left(\left(\beta_{2} D_{2}^{\prime}+\alpha_{2} D_{2}\right) \sin \phi-D_{2} \cos \phi\right) \triangleright \\
& =(\sqrt{J} / 2)\left[Q_{B}\left(\beta_{2} D_{2}^{\prime}+\alpha_{2} D_{2}\right)-Q_{A} D_{2}\right] \\
& =\left(\sqrt{2 \beta_{2} J} / 2\right)\left(R_{36} D_{2}^{\prime}-R_{46} D_{2}\right) .
\end{aligned}
$$

In the last step we have made use of Eq. (22). Combining the above, we find

$$
\begin{aligned}
- & Q\left\langle\Delta \overline{\boldsymbol{J}}^{(h, 1)} \partial_{z J} \delta f^{(M)}\right\rangle \\
& \rightarrow\left(R_{36} D_{2}^{\prime}-R_{46} D_{2}\right) \frac{r_{h}}{\gamma} n_{0 h} w^{\prime}(0) J \partial_{J} F_{h} .
\end{aligned}
$$

The phase-related cooling term is zero since

$-Q\left\langle\Delta \phi^{(h)} \partial_{z \phi} \delta f^{(M)}\right\rangle \propto \int_{-\infty}^{\infty} d k \mathcal{Z}(k) e^{i k z} \partial_{z \phi}\left\langle\delta \hat{n}_{k}^{(M)} \delta f^{(M)}\right\rangle=0$.

Lastly, we turn to the last term in Eq. (33), which is given by

$$
\begin{aligned}
R_{56}^{(h)} & \left\langle\Delta \eta^{(h)} \partial_{z} \delta f^{(M)}\right\rangle \\
& =-R_{56}^{(h)} \frac{r_{h} c}{2 \pi \gamma} \int_{-\infty}^{\infty} d k \mathcal{Z}(k) \partial_{z}\left\langle\delta \hat{n}_{k}^{(M)} \delta f^{(M)}\right\rangle e^{i k z} \\
& =R_{56}^{(h)} \frac{r_{h} c}{2 \pi \gamma} n_{0 h} i F_{h} \int_{-\infty}^{\infty} d k k \mathcal{Z}(k)=-R_{56}^{(h)} \frac{r_{h}}{\gamma} n_{0 h} w^{\prime}(0) F_{h} .
\end{aligned}
$$

Combining all of the terms derived above with Eqs. (32) and (33), we obtain the relation

$$
\begin{aligned}
\frac{\partial F_{h}}{\partial t}= & D_{\eta} \frac{\partial^{2} F_{h}}{\partial \eta^{2}}+D_{\epsilon} \frac{\partial}{\partial J}\left(J \frac{\partial F_{h}}{\partial J}\right) \\
& +w^{\prime}(0) \frac{r_{h}}{T \gamma}\left[-S J \frac{\partial F_{h}}{\partial J}+\left(S-R_{56}^{(h)}\right) \eta \frac{\partial F_{h}}{\partial \eta}-R_{56}^{(h)} F_{h}\right],
\end{aligned}
$$

where the diffusion coefficients $D_{\epsilon}$ and $D_{\eta}$ are defined in Eq. (35). After some minor rearrangement of terms, we arrive at Eq. (34).

where we have only assumed the smallness of $\Delta \eta^{(h)}$, $\Delta \bar{J}^{(h, 1)}$, and $\Delta \phi^{(h)}$ but not of $Q=R_{46} y-R_{36} P-R_{56}^{(h)} \eta=$ $-Q_{0} \eta+\bar{Q}$. Here, $\partial_{2}$ denotes partial differentiation with respect to the second of the arguments of the function under question etc. Thus, the first term we need to calculate is

$T_{1}=R_{56}^{(h)}\left\langle\Delta \eta^{(h)} \partial_{z} \delta f^{(M)}\left(z-Q_{0} \eta+\bar{Q}, \eta, J, \phi-\mu\right)\right\rangle$,

with the hadron energy variation being expressed by the familiar expression of Eq. (28). In order to calculate averages of this kind, we again make use of some relations that follow directly from Eq. (A5), in this case

$$
\begin{aligned}
& \left\langle\delta \hat{n}_{k}^{(M)} \partial_{z} \delta f^{(M)}\left(z-Q_{0} \eta+\bar{Q}, \eta, J, \phi-\mu\right)\right\rangle \\
& \quad=-i k n_{0 h} F_{h} e^{-i k z} e^{i k\left(Q_{0} \eta-\bar{Q}\right)}, \\
& \left\langle\delta \hat{n}_{k}^{(M)} \partial_{2} \delta f^{(M)}\left(z-Q_{0} \eta+\bar{Q}, \eta, J, \phi-\mu\right)\right\rangle \\
& \quad=n_{0 h} \frac{\partial F_{h}}{\partial \eta} e^{-i k z} e^{i k\left(Q_{0} \eta-\bar{Q}\right)}, \\
& \left\langle\delta \hat{n}_{k}^{(M)} \partial_{3} \delta f^{(M)}\left(z-Q_{0} \eta+\bar{Q}, \eta, J, \phi-\mu\right)\right\rangle \\
& \quad=n_{0 h} \frac{\partial F_{h}}{\partial J} e^{-i k z} e^{i k\left(Q_{0} \eta-\bar{Q}\right)}, \\
& \left\langle\delta \hat{n}_{k}^{(M)} \partial_{\phi} \delta f^{(M)}\left(z-Q_{0} \eta+\bar{Q}, \eta, J, \phi-\mu\right)\right\rangle=0 .
\end{aligned}
$$

Thus, we have

$$
\begin{aligned}
T_{1} & =-R_{56}^{(h)} \frac{r_{h} c}{2 \pi \gamma} \int_{-\infty}^{\infty} d k \mathcal{Z}(k) k\left(-i n_{0 h}\right) F_{h} e^{i k Q_{0} \eta} e^{-i k \sqrt{J} R \sin (\phi+\bar{\varepsilon})} \\
& \rightarrow i n_{0 h} F_{h} R_{56}^{(h)} \frac{r_{h} c}{2 \pi \gamma} \int_{-\infty}^{\infty} d k \mathcal{Z}(k) k e^{i k Q_{0} \eta} J_{0}(k R \sqrt{J}), \quad \text { (B4) }
\end{aligned}
$$

where $R=\left(Q_{A}^{2}+Q_{B}^{2}\right)^{1 / 2}$ and $\tan \bar{\varepsilon}=Q_{A} / Q_{B}$ [so that $\left.\bar{Q}=\sqrt{J}\left(Q_{A} \cos \phi+Q_{B} \sin \phi\right)=\sqrt{J} R \sin (\phi+\bar{\varepsilon})\right]$. In the second line of the above result, we have averaged over the betatron phase $\phi$ using the Jacobi-Anger identity, namely

$$
e^{i z \sin \theta}=\sum_{n=-\infty}^{\infty} J_{n}(z) e^{i n \theta}
$$


where $J_{n}(z)$ is a Bessel function of the first kind, so that

$$
\triangleleft e^{i z \sin \theta} \triangleright=J_{0}(z)
$$

Moreover, we can also show that

$$
\triangleleft \cos \theta e^{i(a \cos \theta+b \sin \theta)} \triangleright=i J_{1}\left(\zeta_{0}\right) \sin \theta_{0}
$$

and

$$
\triangleleft \sin \theta e^{i(a \cos \theta+b \sin \theta)} \triangleright=i J_{1}\left(\zeta_{0}\right) \cos \theta_{0},
$$

where $\zeta_{0}=\left(a^{2}+b^{2}\right)^{1 / 2}$ and $\theta_{0}=\tan ^{-1}(a / b)$. We will use these properties in a subsequent calculation. Following a procedure analogous to the one we outlined above, we can deal with the second term of Eq. (B1), i.e.,

$T_{2}=-\left\langle\Delta \eta^{(h)} \partial_{2} \delta f^{(M)}\left(z-Q_{0} \eta+\bar{Q}, \eta, J, \phi-\mu\right)\right\rangle$.

Using the second result in Eq. (B3) (and recalling that $\bar{Q}=\sqrt{J} R \sin (\phi+\bar{\varepsilon}))$, we obtain

$T_{2}=\frac{r_{h} c}{2 \pi \gamma} n_{0 h} \frac{\partial F_{h}}{\partial \eta} \int_{-\infty}^{\infty} d k \mathcal{Z}(k) k e^{i k Q_{0} \eta} J_{0}(k R \sqrt{J})$,

after the betatron phase averaging. The third term of Eq. (B1) is algebraically the most involved one. It is given by

$T_{3}=-\left\langle\Delta \bar{J}^{(h, 1)} \partial_{3} \delta f^{(M)}\left(z-Q_{0} \eta+\bar{Q}, \eta, J, \phi-\mu\right)\right\rangle$

and the result we obtain for it after phase averaging is

$T_{3}=\frac{r_{h} c}{2 \pi \gamma} i n_{0 h} \sqrt{J} \frac{\partial F_{h}}{\partial J} S \int_{-\infty}^{\infty} d k \mathcal{Z}(k) e^{i k Q_{0} \eta} \frac{2 J_{1}(k R \sqrt{J})}{R}$,

where we recall that $S=R_{46} D_{2}-R_{36} D_{2}^{\prime}$. To arrive at this, we have used the previously-mentioned corollaries of the Jacobi-Anger identity [Eqs. (B7) and (B8)], the third result of Eq. (B3) and the definition of $\Delta \bar{J}^{(h, 1)}$ contained in Eq. (26). Finally, the fourth term in the sum of Eq. (B1) is zero, as we can see by combining the final result of Eq. (B3) with the fact that $\Delta \phi^{(h)} \propto \Delta \eta^{(h)}$ [according to Eq. (24)]. Combining the results of this derivation with the previous expressions for the diffusion terms leads to the following form of the kinetic equation:

$$
\begin{aligned}
\frac{\partial F_{h}}{\partial t}= & D_{\eta} \frac{\partial^{2} F_{h}}{\partial \eta^{2}}+D_{\epsilon} \frac{\partial}{\partial J}\left(J \frac{\partial F_{h}}{\partial J}\right) \\
& +\frac{r_{h} c}{2 \pi \gamma T} F_{h} \int_{-\infty}^{\infty} d k \mathcal{Z}(k) i k R_{56}^{(h)} e^{i k Q_{0} \eta} J_{0}(k R \sqrt{J}) \\
& +\frac{r_{h} c}{2 \pi \gamma T} \frac{\partial F_{h}}{\partial \eta} \int_{-\infty}^{\infty} d k \mathcal{Z}(k)\left\{e^{i k Q_{0} \eta} J_{0}(k R \sqrt{J})-1\right\} \\
& +\frac{r_{h} c}{2 \pi \gamma T} i S \sqrt{J} \frac{\partial F_{h}}{\partial J} \int_{-\infty}^{\infty} d k \mathcal{Z}(k) e^{i k Q_{0} \eta} \frac{2 J_{1}(k R \sqrt{J})}{R},
\end{aligned}
$$

where the wake-at-the-origin contribution of Eq. (A7) has been subtracted. To transform the above result into Eq. (41) of the main text, we use the Bessel function identity $J_{1}(\xi)+\xi J_{1}^{\prime}(\xi)=\xi J_{0}(\xi)$ in the form of the property

$$
\frac{2}{R} \frac{d}{d J}\left(\sqrt{J} J_{1}(k R \sqrt{J})\right)=k J_{0}(k R \sqrt{J}) .
$$

\section{APPENDIX C: 1D SPACE CHARGE FORCE FOR BEAMS WITH AN ELLIPTICAL CROSS SECTION}

For our analysis, the effect of space charge — encompassing both the electromagnetic interaction between the hadron and electron beams in the modulator/kicker sections and the selfinteraction in a possible plasma amplification module-is of crucial importance. In our simplified model, space charge is described in a one-dimensional framework by subdividing each beam longitudinally into a collection of infinitely thin segments (slices), which are characterized by a common charge and a prescribed transverse profile for the charge density. A similar approach was followed in [10], assuming an axially symmetric, Gaussian profile. Since the beams we are dealing with are-generally speaking_not round, we instead choose a Gaussian profile with an elliptical cross section. In the rest frame of the beam (or in the rest frame of two beams copropagating with the same velocity), the interaction between two slices is essentially an electrostatics problem. Thus, we start our analysis by considering the force between two slices with charges $Q$ and $Q_{0}$-belonging, in general, to different beams - that remain at rest, separated by a longitudinal distance $z$. We note that, in this Appendix, the symbols $Q$ and $Q_{0}$ are unrelated to the quantities introduced in Eq. (21). The surface charge densities for the two slices are given by

$$
\begin{gathered}
\rho_{0}(x, y)=\frac{Q_{0}}{2 \pi \Sigma_{x 0} \Sigma_{y 0}} \exp \left(-\frac{x_{0}^{2}}{2 \Sigma_{x 0}^{2}}\right) \exp \left(-\frac{y_{0}^{2}}{2 \Sigma_{y 0}^{2}}\right), \\
\rho(x, y)=\frac{Q}{2 \pi \Sigma_{x} \Sigma_{y}} \exp \left(-\frac{x^{2}}{2 \Sigma_{x}^{2}}\right) \exp \left(-\frac{y^{2}}{2 \Sigma_{y}^{2}}\right),
\end{gathered}
$$


where $\left(x_{0}, y_{0}\right)$ and $(x, y)$ denote the transverse positions for the two slices while $\Sigma_{x 0}, \Sigma_{y 0}$ and $\Sigma_{x}, \Sigma_{y}$ are the corresponding rms beam sizes. The two charge density functions satisfy $\int_{-\infty}^{\infty} d x \int_{-\infty}^{\infty} d y \rho(x, y)=Q$ and $\int_{-\infty}^{\infty} d x_{0} \int_{-\infty}^{\infty} d y_{0} \rho_{0}\left(x_{0}, y_{0}\right)=Q_{0}$. The longitudinal electric field at point $(x, y, z)$ due to the charge $Q_{0}$ is

$$
\begin{aligned}
E_{z} & =-\frac{\partial V}{\partial z} \\
& =-\frac{\partial}{\partial z} \int_{-\infty}^{\infty} d x_{0} \int_{-\infty}^{\infty} d y_{0} \frac{\rho_{0}\left(x_{0}, y_{0}\right)}{\left[\left(x-x_{0}\right)^{2}+\left(y-y_{0}\right)^{2}+z^{2}\right]^{1 / 2}} \\
& =\int_{-\infty}^{\infty} d x_{0} \int_{-\infty}^{\infty} d y_{0} \frac{z \rho_{0}\left(x_{0}, y_{0}\right)}{\left[\left(x-x_{0}\right)^{2}+\left(y-y_{0}\right)^{2}+z^{2}\right]^{3 / 2}},
\end{aligned}
$$

where $V$ is the electrostatic potential. As a result, the longitudinal force between the two slices is given by

$$
\begin{aligned}
F_{z}= & \int_{-\infty}^{\infty} d x \int_{-\infty}^{\infty} d y \rho(x, y) E_{z}(x, y, z) \\
= & z \int_{-\infty}^{\infty} d x \int_{-\infty}^{\infty} d y \rho(x, y) \int_{-\infty}^{\infty} d x_{0} \int_{-\infty}^{\infty} d y_{0} \rho_{0}\left(x_{0}, y_{0}\right) \\
& \times\left[\left(x-x_{0}\right)^{2}+\left(y-y_{0}\right)^{2}+z^{2}\right]^{-3 / 2} .
\end{aligned}
$$

To facilitate further analysis, we use the identity

$$
\frac{1}{R_{0}^{3}}=\frac{4}{\sqrt{\pi}} \int_{0}^{\infty} d \lambda \lambda^{2} \exp \left(-\lambda^{2} R_{0}^{2}\right)
$$

with $R_{0} \rightarrow\left[\left(x-x_{0}\right)^{2}+\left(y-y_{0}\right)^{2}+z^{2}\right]^{1 / 2}$ in order to transform Eq. (C4) into

$$
\begin{aligned}
F_{z}= & \frac{4 z}{\sqrt{\pi}} \int_{0}^{\infty} d \lambda \lambda^{2} \exp \left(-\lambda^{2} z^{2}\right) \int_{-\infty}^{\infty} d x \int_{-\infty}^{\infty} d y \rho(x, y) \\
& \times \int_{-\infty}^{\infty} d x_{0} \int_{-\infty}^{\infty} d y_{0} \rho_{0}\left(x_{0}, y_{0}\right) \\
& \times \exp \left(-\lambda^{2}\left[\left(x-x_{0}\right)^{2}+\left(y-y_{0}\right)^{2}\right]\right) .
\end{aligned}
$$

Substituting Eqs. (C1) and (C2) into Eq. (C6) yields an expression for the force after some straightforward Gaussian integration. The end result is

$$
\begin{aligned}
F_{z}= & \frac{4 Q Q_{0} z}{\sqrt{\pi}} \int_{0}^{\infty} d \lambda \lambda^{2} \\
& \times \frac{\exp \left(-\lambda^{2} z^{2}\right)}{\sqrt{1+2 \lambda^{2} \Sigma_{x}^{2}\left(1+r_{x}^{2}\right)} \sqrt{1+2 \lambda^{2} \Sigma_{y}^{2}\left(1+r_{y}^{2}\right)}},
\end{aligned}
$$

where $r_{x}=\Sigma_{x 0} / \Sigma_{x}$ and $r_{y}=\Sigma_{y 0} / \Sigma_{y}$. For simplification, in what follows, we will assume full overlap between the two slices, so $\Sigma_{x}=\Sigma_{x 0}, \quad \Sigma_{y}=\Sigma_{y 0}$ and $r_{x}=r_{y}=1$. This assumption is also adopted in the main text. The new expression for the force can be written as

$$
F_{z}=\frac{Q Q_{0}}{\Sigma_{x}^{2}} \Phi\left(\frac{z}{\Sigma_{x}}, r\right)
$$

where $r=\Sigma_{y} / \Sigma_{x}$ is the aspect ratio of the slices and the scaled interaction function $\Phi$ is given by

$$
\Phi(\hat{z}, r)=\frac{4 \hat{z}}{\sqrt{\pi}} \int_{0}^{\infty} d \hat{\lambda} \hat{\lambda}^{2} \frac{\exp \left(-\hat{\lambda}^{2} \hat{z}^{2}\right)}{\sqrt{1+4 \hat{\lambda}^{2}} \sqrt{1+4 r^{2} \hat{\lambda}^{2}}} .
$$

To obtain the force in the laboratory frame, it is only necessary to set $z \rightarrow \gamma z$, where $\gamma$ is the relativistic factor of the beam. This is so because a Lorentz transformation in the $z$-direction (the direction of the beam velocity) does not alter $F_{z}$ or the transverse sizes of the two slices. It does, however, result in a length contraction given by $\Delta l^{(\text {lab })}=\Delta l^{(\text {beam })} / \gamma$. Thus the longitudinal force in the $l a b$ frame is

$$
F_{z}=\frac{Q Q_{0}}{\Sigma_{x}^{2}} \Phi\left(\frac{\gamma z}{\Sigma_{x}}, r\right)
$$

Setting $Q=Z e$ and $Q_{0}=-e$, we recover Eq. (50) of the main text.

The interaction function $\Phi$, as defined by Eq. (C9), is antisymmetric with respect to $\hat{z}$ (see left panel of Fig. 2), so that

$$
\Phi(-\hat{z}, r)=-\Phi(\hat{z}, r) .
$$

As a direct result of this we have $\Phi(\hat{z}=0, r)=0$. Moreover, $\Phi$ satisfies the relation

$$
\Phi(\hat{z}, 1 / r)=r^{2} \Phi(r \hat{z}, r) .
$$

The latter property can also be deduced from Eq. (C10) in the following way: selecting which of the transverse dimensions to label $x$ and which $y$ is, in fact, arbitrary and should not affect the actual value of the force between the two slices. As a result, the RHS of Eq. (C10) should remain invariant under the transformation $\Sigma_{x} \rightarrow \Sigma_{y}$ and $\Sigma_{y} \rightarrow \Sigma_{x}$, so that $\Phi\left(\gamma z / \Sigma_{x}, \Sigma_{y} / \Sigma_{x}\right) / \Sigma_{x}^{2}=\Phi\left(\gamma z / \Sigma_{y}, \Sigma_{x} / \Sigma_{y}\right) / \Sigma_{y}^{2}$. Setting $\gamma z / \Sigma_{x} \rightarrow \hat{z}$ and $\Sigma_{x} / \Sigma_{y} \rightarrow \hat{r}$, we can see that this is essentially identical to the relation we seek to prove. In the limit $\hat{z} \rightarrow \infty$, $\Phi(\hat{z}, r) \rightarrow 1 / \hat{z}^{2}$ so the force is $F_{z}=Q Q_{0} /\left(\gamma^{2} z^{2}\right)$. This corresponds to the case where the slices are so far apart that they can be treated as point charges. On the other hand, when $\hat{z} \approx 0^{+}$, we have $\Phi(\hat{z}, r) \approx 1 /(2 r)$.

From a detailed numerical analysis of Eq. (C9), we can also obtain a very useful fitting formula for the interaction function $\Phi$, namely

$$
\Phi(\hat{z}, r) \approx \bar{b}(r) \frac{1+\exp (-\bar{a}(r) \hat{z})}{2+\bar{c}(r) \hat{z}+\bar{b}(r) \hat{z}^{2}},
$$

where 


$$
\begin{aligned}
\bar{a}(r) & =1.628 r^{-0.526}, \\
\bar{b}(r) & =0.499 r^{-0.998}, \\
\bar{c}(r) & =0.0919 / r^{1.794}+0.0583 r^{1.297} .
\end{aligned}
$$

This formula is meant to be used for $\hat{z}>0$ (and has been verified for $0.25 \leq r \leq 2.5$ ). For $\hat{z}<0$, we can reconstruct the function $\Phi$ by means of Eq. (C11). Furthermore, we can readily verify that Eq. (C13) reproduces the expected behavior of $\Phi$ for $\hat{z} \rightarrow 0^{+}$and $\hat{z} \rightarrow \infty$. It also-to a good approximation-satisfies Eq. (C12).

Lastly, we note that, for the case of slices with a circular cross section $(r=1)$, Eq. (C9) reduces to

$\Phi(\hat{z}, r=1)=-\frac{2}{\sqrt{\pi}} \frac{d}{d \hat{z}} \int_{0}^{\infty} d \hat{\lambda} \frac{\exp \left(-\hat{\lambda}^{2} \hat{z}^{2}\right)}{1+4 \hat{\lambda}^{2}} \equiv-\frac{2}{\sqrt{\pi}} \frac{d \tilde{I}}{d \hat{z}}$.

The integral $\tilde{I}$ can be shown to be equal to $(\pi / 4) \exp \left(\hat{z}^{2} / 4\right) \times$ $\operatorname{erfc}(|\hat{z}| / 2)$, where $\operatorname{erfc}(x)=(2 / \sqrt{\pi}) \int_{x}^{\infty} d t \exp \left(-t^{2}\right)$ is the complementary error function. Substituting this result back into Eq. (C15), we obtain

$\Phi(\hat{z}, r=1)=\frac{\hat{z}}{2|\hat{z}|}-\frac{\sqrt{\pi} \hat{z}}{4} \exp \left(\hat{z}^{2} / 4\right) \operatorname{erfc}(|\hat{z}| / 2)$,

which is the analytical expression that we used in our earlier, one-dimensional treatment of MBEC (Refs. [5,6]). Furthermore, the same relation has been discussed in [11].
[1] D. Ratner, Microbunched Electron Cooling for HighEnergy Hadron Beams, Phys. Rev. Lett. 111, 084802 (2013).

[2] Y. S. Derbenev, On possibilities of fast cooling of heavy particle beams, AIP Conf. Proc. 253, 103 (1992).

[3] V. N. Litvinenko and Y.S. Derbenev, Coherent Electron Cooling, Phys. Rev. Lett. 102, 114801 (2009).

[4] V. N. Litvinenko, G. Wang, D. Kayran, Y. Jing, J. Ma, and I. Pinayev, Technical Report, 2018, arXiv:1802.08677.

[5] G. Stupakov, Cooling rate for microbunched electron cooling without amplification, Phys. Rev. Accel. Beams 21, 114402 (2018).

[6] G. Stupakov and P. Baxevanis, Microbunched electron cooling with amplification cascades, Phys. Rev. Accel. Beams 22, 034401 (2019).

[7] V. Lebedev, Optical stochastic cooling, ICFA Beam Dyn. Newslett. 65, 100 (2014).

[8] G. Stupakov and P. Baxevanis, in Proceedings of the 10th International Particle Accelerator Conference (Melbourne, Australia, 2019), p. 814.

[9] M. Babzien, I. Ben-Zvi, I. Pavlishin, I. V. Pogorelsky, V.E. Yakimenko, A. A. Zholents, and M.S. Zolotorev, Optical stochastic cooling for RHIC using optical parametric amplification, Phys. Rev. Accel. Beams 7, 012801 (2004).

[10] M. Dohlus, E. A. Schneidmiller, and M. V. Yurkov, Generation of attosecond soft $\mathrm{x}$-ray pulses in a longitudinal space charge amplifier, Phys. Rev. Accel. Beams 14, 090702 (2011).

[11] G. Geloni, E. Saldin, E. Schneidmiller, and M. Yurkov, Longitudinal wake field for an electron beam accelerated through an ultrahigh field gradient, Nucl. Instrum. Methods Phys. Res., Sect. A 578, 34 (2007). 